Article

\title{
On Boundary Layer Expansions for a Singularly Perturbed Problem with Confluent Fuchsian Singularities
}

\section{Stephane Malek}

Laboratoire Paul Painlevé, University of Lille, 59655 Villeneuve d'Ascq CEDEX, France; stephane.malek@univ-lille.fr

Received: 20 December 2019; Accepted: 26 January 2020; Published: 4 February 2020

check for updates

\begin{abstract}
We consider a family of nonlinear singularly perturbed PDEs whose coefficients involve a logarithmic dependence in time with confluent Fuchsian singularities that unfold an irregular singularity at the origin and rely on a single perturbation parameter. We exhibit two distinguished finite sets of holomorphic solutions, so-called outer and inner solutions, by means of a Laplace transform with special kernel and Fourier integral. We analyze the asymptotic expansions of these solutions relatively to the perturbation parameter and show that they are (at most) of Gevrey order 1 for the first set of solutions and of some Gevrey order that hinges on the unfolding of the irregular singularity for the second.
\end{abstract}

Keywords: asymptotic expansion; Borel-Laplace transform; Fourier transform; initial value problem; formal power series; linear integro-differential equation; partial differential equation; singular perturbation

2010 MSC: 35R10; 35C10; 35C15; 35C20

\section{Introduction}

In paper [1], we considered families of singular PDEs of the next type

$$
\begin{aligned}
Q\left(\partial_{z}\right) y(t, z, \epsilon)= & Q_{1}\left(\partial_{z}\right) y(t, z, \epsilon) Q_{2}\left(\partial_{z}\right) y(t, z, \epsilon)+\epsilon^{\delta_{D}} t_{t} \delta_{D}(k+1) \partial_{t}^{\delta_{D}} R_{D}\left(\partial_{z}\right) y(t, z, \epsilon) \\
& +\sum_{l=1}^{D-1} \epsilon^{\Delta_{l}} t^{d_{l}} \partial_{t}^{\delta_{l}} R_{l}\left(\partial_{z}\right) y(t, z, \epsilon)+f(t, z, \epsilon)
\end{aligned}
$$

where $D, k \geq 1, \Delta_{l}, d_{l}, \delta_{l}, \delta_{D} \geq 0$ are integers, $Q(X), Q_{1}(X), Q_{2}(X), R_{D}(X), R_{l}(X), 1 \leq l \leq D-1$ are polynomials with complex coefficients, and the forcing term $f(t, z, \epsilon)$ represents a bounded holomorphic function regarding the time $t$, the parameter $\epsilon$ in the vicinity of the origin in $\mathbb{C}$ and relatively to $z$ on a horizontal strip $H_{\beta}=\{z \in \mathbb{C} /|\operatorname{Im}(z)|<\beta\}$ of width $\beta>0$. Under the assumption that

$$
\Delta_{l} \geq d_{l}-\delta_{l}, d_{l}>\delta_{l}(k+1)
$$

for $1 \leq l \leq D-1$, these equations can be rewritten in the next manner

$$
\begin{aligned}
Q\left(\partial_{z}\right) y(t, z, \epsilon)= & Q_{1}\left(\partial_{z}\right) y(t, z, \epsilon) Q_{2}\left(\partial_{z}\right) y(t, z, \epsilon)+\left(\epsilon^{k} t^{k+1} \partial_{t}\right)^{\delta_{D}} R_{D}\left(\partial_{z}\right) y(t, z, \epsilon) \\
& +P\left(t, \epsilon, \epsilon^{k} t^{k+1} \partial_{t}, \partial_{z}\right) y(t, z, \epsilon)+f(t, z, \epsilon)
\end{aligned}
$$

where $P\left(t, \epsilon, V_{1}, V_{2}\right)$ is polynomial in its arguments $V_{1}, V_{2}$ with holomorphic coefficients in $t, \epsilon$. These equations can be expressed solely in terms of the basic singularly perturbed differential operator of irregular type $\epsilon^{k} t^{k+1} \partial_{t}$ with so-called rank $k$ acting on time. 
We have shown that these equations possess families of holomorphic solutions $y_{p}(t, z, \epsilon)$ that can be expressed through Laplace transforms of order $k$ and Fourier inverse integrals

$$
y_{p}(t, z, \epsilon)=\frac{k}{(2 \pi)^{1 / 2}} \int_{-\infty}^{+\infty} \int_{L_{\gamma p}} \omega_{p}(u, m, \epsilon) \exp \left(-\left(\frac{u}{\epsilon t}\right)^{k}\right) e^{i z m} \frac{d u}{u} d m
$$

along halflines $L_{\gamma_{p}}=[0,+\infty) e^{\sqrt{-1} \gamma_{p}}$ enclosed in a suitable unbounded sector $S_{d_{p}}$ with bisecting direction $d_{p} \in \mathbb{R}$, where $\omega_{p}$ stands for a holomorphic function near the origin and with exponential growth regarding $u$ on $S_{d_{p}}$, continuous regarding $m$ on $\mathbb{R}$ suffering exponential decay and relying analytically on the perturbation parameter $\epsilon$ on a punctured disc $D\left(0, \epsilon_{0}\right) \backslash\{0\}$. These functions $y_{p}$ are bounded holomorphic on domains $\mathcal{T} \times H_{\beta} \times \mathcal{E}_{p}$, for some proper chosen bounded sector $\mathcal{T}$ with vertex at 0 and $\underline{\mathcal{E}}=\left\{\mathcal{E}_{p}\right\}_{0 \leq p \leq l-1}$ a set of bounded sectors whose union covers a full neighborhood of 0 in $\mathbb{C}^{*}$ called a good covering (see Definition 4 ). Furthermore, they share a common asymptotic expansion $\hat{y}(t, z, \epsilon)=\sum_{k \geq 0} y_{k}(t, z) \epsilon^{k}$ that turns out to be of Gevrey order $1 / k$ with respect to the perturbation parameter $\epsilon$, meaning that two constants $C_{p}, K_{p}>0$ can be found with

$$
\sup _{t \in \mathcal{T}, z \in H_{\beta}}\left|y_{p}(t, z, \epsilon)-\sum_{k=0}^{n-1} y_{k}(t, z) \epsilon^{k}\right| \leq C_{p}\left(K_{p}\right)^{n} \Gamma\left(1+\frac{n}{k}\right)|\epsilon|^{n}
$$

for all $n \geq 1$, provided that $\epsilon \in \mathcal{E}_{p}$.

In this work, we depart from Equation (2) in the special case $k=1$. We unfold the operator $\epsilon t^{2} \partial_{t}$ into a family of singular operators

$$
\mathcal{D}_{\epsilon, \alpha}\left(\partial_{t}\right)=\left(\epsilon t^{2}-\epsilon^{\alpha}\right) \partial_{t}
$$

depending on a parameter $\alpha \geq 3$, assumed to be an odd natural number, which are now Fuchsian at the points $t= \pm \epsilon^{\frac{\alpha-1}{2}}$. Notice that these singular points merge to 0 as $\epsilon$ borders the origin. In other words, we focus on equations of the next form

$$
\begin{aligned}
Q\left(\partial_{z}\right) u(t, z, \epsilon)= & Q_{1}\left(\partial_{z}\right) u(t, z, \epsilon) Q_{2}\left(\partial_{z}\right) u(t, z, \epsilon)+\left(\mathcal{D}_{\epsilon, \alpha}\left(\partial_{t}\right)\right)^{\delta_{D}} R_{D}\left(\partial_{z}\right) u(t, z, \epsilon) \\
& +P\left(t, z, \epsilon, \mathcal{D}_{\epsilon, \alpha}\left(\partial_{t}\right), \partial_{z}\right) y(t, z, \epsilon)+f(t, z, \epsilon)
\end{aligned}
$$

where $P$ is analytic in $t, z, \epsilon$ and polynomial in the operators $\mathcal{D}_{\epsilon, \alpha}\left(\partial_{t}\right), \partial_{z}$ and $f$ is a forcing term as above. The coefficients of $P$ and the forcing term $f$ represent bounded analytic functions in $z$ and $\epsilon$ and, as a new feature, involve a well-chosen logarithmic function in time variable (described in (21)) that is adapted to our problem. They belong to a class of functions that is more restrictive than the one considered in our previous Equation (2). However, notice that according to Lemma 9 and 10, these functions are good approximations of general bounded analytic functions of the form $h(\epsilon t, z, \epsilon)$ on appropriate domains in time $t$, for $z \in H_{\beta}$, provided that $\epsilon$ is close enough to 0 .

It is worthwhile noting that PDEs with Fuchsian singularities and logarithmic coefficients appear in several recent works by different authors. Specifically, in papers [2,3], Jose Ernie C. Lope investigates existence and uniqueness properties of solutions to linear Fuchsian equations with the shape

$$
\left(t \partial_{t}\right)^{m} u(t, x)=G\left(t, x,\left(\left(\mu(t) \partial_{x}\right)^{\alpha}\left(t \partial_{t}\right)^{j} u(t, x)\right)_{(\alpha, j) \in I_{m}}\right)
$$

where $G\left(t, x,\left(u_{\alpha, j}\right)_{(\alpha, j) \in I_{m}}\right)$ stands for a linear form in $u_{\alpha, j}$, holomorphic in $x$ on a bounded domain of $\mathbb{C}^{n}$ and continuous in $t$ on $[0, T], T>0$ and where the so-called weight function $\mu(t)$ may involve functions like $1 /(-\log (t))^{k+1}$ provided that $k>0$. In paper [4], Hidetoshi Tahara and Hideshi Yamane solve nonlinear equations of the form

$$
\left(t \partial_{t}\right)^{m} u(t, x)=H\left(t, x,\left(\left(t \partial_{t}\right)^{j} \partial_{x}^{\alpha} u(t, x)\right)_{(j, \alpha) \in J_{m}}\right)
$$


of Fuchsian type at $t=0$, where $H$ represents a bounded holomorphic function whose coefficients includes powers of $\log (t)$. In the study [5], Hideshi Yamane constructs solutions to nonlinear wave equations that blow up along prescribed noncharacteristic hypersurfaces using the so-called Fuchsian reduction method introduced by S. Kichenassamy which transforms the initial problem into a Fuchsian PDE which, in general, contains logarithmic terms, see the excellent textbook [6] for a reference.

The idea of considering such special types of confluence (3) stems from a work by M. Klimes, see [7], where nonlinear differential systems with irregular singularity at $x=0$

$$
x^{2} \underline{y}^{\prime}(x)=M \underline{y}(x)+f(x, \underline{y}(x))
$$

with invertible matrix $M$ of dimension $n \geq 1$ and unknown vector function

$$
\underline{y}(x)=\left(y_{1}(x), \ldots, y_{n}(x)\right)
$$

where $f$ stands for an analytic function near the origin in $\mathbb{C}^{n+1}$, are unfolded into a deformed complex parameter depending differential system

$$
\left(x^{2}-\epsilon\right) \partial_{x} \underline{y}(x, \epsilon)=M(\epsilon) \underline{y}(x, \epsilon)+f(x, \epsilon, \underline{y}(x))
$$

with Fuchsian singularities at $x= \pm \epsilon^{1 / 2}$ that coalesce to the origin as $\epsilon$ tends to 0 . This paper [7] can be seen as a continuation of the contribution by B. Sternin and V. Shatalov [8] who focused on linear scalar ODEs with holomorphic coefficients of the form

$$
H\left(x, x(x+\epsilon) \frac{d}{d x}\right) y(x)=0
$$

for a small complex parameter $\epsilon$.

Further important recent works on confluence of singularities that are somehow related to our study need to be mentioned. In paper [9], Reinhard Schäfke expounds the confluence of so-called hypergeometric systems of Okubo type to Birkhoff normal forms. This result has been extended to general linear systems of ODEs in two seminal papers $[10,11]$ by Alexey Glutsyuk that describe the convergence of monodromy data of well-chosen fundamental solutions to the Stokes matrices in the confluence process. More recently in [12], Tsvetana Stoyanova has studied particular cases of $[10,11]$ and obtained explicit formulas for the solutions of an unfolding of a third order linear scalar ODE with irregular singularity at the origin. Confluence under the additional constraint of isomonodromic deformation has been investigated for linear systems of ODEs for Fuchsian singularities by Andrey Bolibrukh, ref. [13] and later extended to the case of irregular singularities by Yulia Bibilo in [14]. Confluence of Fuchsian singularities for linear scalar ODEs has also been undertaken from the point of view of the so-called index of rigidity by Toshio Oshima in [15] and extensions to Pfaffian systems of PDEs are investigated using the middle convolution procedure in [16]. In the series of papers [17-20], the authors classify and provide normal forms for generic unfolding of nonresonant linear systems of ODEs with irregular singularity at the origin. In [21], confluent non-autonomous Hamiltonian systems are studied and applications to the degeneration processes for the famous Painleve sixth equation are discussed. We mention also the novel work [22] by Jorge Mozo-Fernández and Reinhard Schäfke where confluence of Fuchsian and more general singularities can be unified in a general theory through the concept of summability regarding an analytic germ.

From a wider standpoint, our study falls in the asymptotic theory of singularly perturbed problems. For a far-reaching introduction and foundation of this active domain of research we may refer to the monograph [23] by S.A. Lomov. For other recent textbooks providing useful examples we can suggest [24-27]. 
In this paper, our aim is the study of the effect of the small perturbation $\epsilon^{\alpha}$ in the singular operators $\mathcal{D}_{\epsilon, \alpha}\left(\partial_{t}\right)$ on the asymptotic properties of (well-chosen) holomorphic solutions to our problem (4) in comparison to the ones of the departing problem (2).

One first major change in our new setting is the fact that the holomorphic solutions we build up for (4) (see Theorems 1 and 2) are no longer defined on a full sector $\mathcal{T}$ centered at 0 in time $t$. Instead, we manufacture two specific families of analytic functions. The elements of the first one, we call the outer solutions, $u_{p}^{\text {out }}(t, z, \epsilon), 0 \leq p \leq \iota-1$, are defined in time $t$ on a fixed sectorial annulus $\mathcal{A}$ (see (65)) that does not rely on $\epsilon$ and is confined apart of the origin. The second family is comprised by so-called inner solutions, $u_{p}^{\text {in }}(t, z, \epsilon), 0 \leq p \leq \eta-1$ that are built up on a domain in time $\mathcal{T}_{\epsilon}$ (see (81)) that hinges on $\epsilon$, stays close to the Fuchsian singularities $\pm \epsilon^{\frac{\alpha-1}{2}}$ and borders the origin as $\epsilon$ comes close to 0 .

The second substantial difference dwells on the parametric asymptotic expansions of these solutions. Specifically, provided that $t$ is kept (at least) at some fixed distance of the origin in $\mathcal{A}$, the outer solutions $u_{p}^{\text {out }}(t, z, \epsilon)$ have similar asymptotic expansions in $\epsilon$ as the one observed for the analytic solutions $y_{p}(t, z, \epsilon)$ of (2) (in the case $k=1$ ), indeed they are (at most) of Gevrey order 1. On the other hand, when $t$ is next to the Fuchsian singularities $\pm \epsilon^{\frac{\alpha-1}{2}}$ on $\mathcal{T}_{\epsilon}$, the Gevrey order of the asymptotic expansions relatively to $\epsilon$ of the inner solutions $u_{p}^{\text {in }}(t, z, \epsilon)$ turns out to be sensitive on the small perturbation $\epsilon^{\alpha}$ in (3) and becomes (at most) equal to $\frac{2}{\alpha+1}$, see Theorem 3 .

We undertake the main problem (4) by following a closely related approach to the one used [7] and which actually originates from [8]. Specifically, we exhibit a first family of bounded holomorphic solutions to (4), we name outer solutions, in the form of special Laplace transforms and Fourier integrals

$$
(t, z, \epsilon) \mapsto u_{p}^{\text {out }}(t, z, \epsilon)=\frac{1}{(2 \pi)^{1 / 2}} \int_{-\infty}^{+\infty} \int_{L_{\gamma_{p}^{\text {out }}}} W_{p}^{\text {out }}(\tau, m, \epsilon) \exp (\tau a(\epsilon t, \epsilon)) e^{i z m} \frac{d \tau}{\tau} d m
$$

defined on domains $\mathcal{A} \times H_{\beta} \times \mathcal{E}_{p}^{\text {out }}$, where $\mathcal{E}_{p}^{\text {out }}$ are bounded sectors that belong to a well-chosen good covering $\underline{\mathcal{E}}^{\text {out }}$, see Theorem 1 . We distinguish a second set of so-called inner solutions also expressed in a similar manner as the outer functions, namely

$$
(t, z) \mapsto u_{p}^{\text {in }}(t, z, \epsilon)=\frac{1}{(2 \pi)^{1 / 2}} \int_{-\infty}^{+\infty} \int_{L_{\gamma_{p}}^{\text {in }}} W_{p}^{\text {in }}(\tau, m, \epsilon) \exp (\tau a(\epsilon t, \epsilon)) e^{i z m} \frac{d \tau}{\tau} d m
$$

on domains $\mathcal{T}_{\epsilon} \times H_{\beta}$, provided that $\epsilon \in \mathcal{E}_{p}^{\text {in }}$ for a collection of bounded sectors $\mathcal{E}_{p}^{\text {in }}$ which constitutes a convenient good covering $\underline{\mathcal{E}}^{\text {in }}$, see Theorem 2 . In both constructions, the function $a(T, \epsilon)$ isproperly selected in a way that the differential operator $\mathcal{D}_{\epsilon, \alpha}\left(\partial_{t}\right)$ acts on $u_{p}^{\text {out/in }}$ as a multiplication by $\tau$ on the Borel map $W_{p}^{\text {out } / \text { in }}(\tau, m, \epsilon)$, see $(20)$.

Notice that in [7], bilateral Laplace transforms are introduced which operate on Borel maps that are holomorphic on strips. The restricted class of admissible coefficients for our main Equation (4) allows us to obtain Borel maps that are defined on unbounded sectors in the Borel plane. We can merely use the classical single side Laplace transforms to construct our solutions (5), (6) and apply similar constructions as in our previous study [1].

Our paper is organized as follows: In Section 2, we set forth the main problem of the work (11) and we depict the strategy which leads to its resolution. Specifically, we search for a solution expressed through special Laplace and Fourier transform (19). The main achievement of this section is the statement of a convolution Equation (29) fulfilled by the Borel map W of the solution. In Section 3, we solve the convolution problem (29) within Banach spaces of holomorphic and continuous functions with exponential growth on unbounded sectors in the Borel plane and exponential decay in real phase. In Section 4.1, we build up a family of outer solutions defined on a covering set of sectors in the perturbation parameter $\epsilon$ and on a fixed sectorial annulus in time (Theorem 1). In Section 4.2, a collection of inner solutions is exhibited which are defined relatively to $\epsilon$ on a good covering in $\mathbb{C}^{*}$ and regarding time on a domain that remains close to the moving Fuchsian singularities of the main 
problem. In the last Section 4.3, we analyze the asymptotic behavior of the latter two distinguished families of outer/inner solutions, by means of the classical Ramis-Sibuya approach (Theorem 3).

\section{Layout of the Main Problem and Related Auxiliary Equations}

\subsection{Short Review of Fourier transforms}

We restate the definition of some family of Banach spaces mentioned in [1].

Definition 1. Let $\beta, \mu \in \mathbb{R}$. We set $E_{(\beta, \mu)}$ as the vector space of continuous functions $h: \mathbb{R} \rightarrow \mathbb{C}$ such that

$$
\|h(m)\|_{(\beta, \mu)}=\sup _{m \in \mathbb{R}}(1+|m|)^{\mu} \exp (\beta|m|)|h(m)|
$$

is finite. The space $E_{(\beta, \mu)}$ endowed with the norm $\|\cdot\|_{(\beta, \mu)}$ becomes a Banach space.

We remind the reader the definition of the inverse Fourier transform acting on the latter Banach spaces and some of its handy formulas relative to derivation and convolution product as stated in [1].

Definition 2. Let $f \in E_{(\beta, \mu)}$ with $\beta>0, \mu>1$. The inverse Fourier transform of $f$ is given by

$$
\mathcal{F}^{-1}(f)(x)=\frac{1}{(2 \pi)^{1 / 2}} \int_{-\infty}^{+\infty} f(m) \exp (i x m) d m
$$

for all $x \in \mathbb{R}$. The function $\mathcal{F}^{-1}(f)$ extends to an analytic bounded function on the strips

$$
H_{\beta^{\prime}}=\left\{z \in \mathbb{C} /|\operatorname{Im}(z)|<\beta^{\prime}\right\} .
$$

for all given $0<\beta^{\prime}<\beta$.

(a) Define the function $m \mapsto \phi(m)=i m f(m)$ which belongs to the space $E_{(\beta, \mu-1)}$. Then, the next identity

$$
\partial_{z} \mathcal{F}^{-1}(f)(z)=\mathcal{F}^{-1}(\phi)(z)
$$

occurs on $H_{\beta^{\prime}}$ for $0<\beta^{\prime}<\beta$.

(b) Take $g \in E_{(\beta, \mu)}$ and set

$$
\psi(m)=\frac{1}{(2 \pi)^{1 / 2}} \int_{-\infty}^{+\infty} f\left(m-m_{1}\right) g\left(m_{1}\right) d m_{1}
$$

as the convolution product of $f$ and $g$. Then, $\psi$ belongs to $E_{(\beta, \mu)}$ and moreover,

$$
\mathcal{F}^{-1}(f)(z) \mathcal{F}^{-1}(g)(z)=\mathcal{F}^{-1}(\psi)(z)
$$

for all $z \in H_{\beta^{\prime}}$, for $0<\beta^{\prime}<\beta$.

\subsection{Statement of the Main Problem}

Let $D \geq 1, \delta_{D} \geq 2$ be natural numbers, $\alpha \geq 3$ be an odd natural number, $c_{1,2} \in \mathbb{C}^{*}$ a non-vanishing complex number and $\epsilon \in \mathbb{C}^{*}$ a complex parameter. Let $\delta_{l} \geq 0$ be non-negative integers with

$$
\delta_{l}<\delta_{l+1}
$$

for all $0 \leq l \leq D-1$. We define the differential operator

$$
\mathcal{D}_{\epsilon, \alpha}\left(\partial_{t}\right)=\left(\epsilon t^{2}-\epsilon^{\alpha}\right) \partial_{t}
$$


and for any integer $l \geq 1$ we denote $\left(\mathcal{D}_{\epsilon, \alpha}\left(\partial_{t}\right)\right)^{l}$ the iteration of order $l$ of the operator $\mathcal{D}_{\epsilon, \alpha}\left(\partial_{t}\right)$.

We set $Q(X), Q_{1}(X), Q_{2}(X), R_{D}(X), R_{l}(X), 0 \leq l \leq D-1$ as polynomials such that

$$
\begin{gathered}
\operatorname{deg}(Q) \geq \operatorname{deg}\left(R_{D}\right) \geq \operatorname{deg}\left(R_{l}\right), \operatorname{deg}\left(R_{D}\right) \geq \max \left(\operatorname{deg}\left(Q_{1}\right), \operatorname{deg}\left(Q_{2}\right)\right), \\
Q(i m) \neq 0, R_{D}(i m) \neq 0
\end{gathered}
$$

for all $m \in \mathbb{R}$. We state the main initial value problem of our study

$$
\begin{aligned}
Q\left(\partial_{z}\right) u(t, z, \epsilon)= & c_{1,2} Q_{1}\left(\partial_{z}\right) u(t, z, \epsilon) Q_{2}\left(\partial_{z}\right) u(t, z, \epsilon)+\left(\mathcal{D}_{\epsilon, \alpha}\left(\partial_{t}\right)\right)^{\delta_{D}} R_{D}\left(\partial_{z}\right) u(t, z, \epsilon) \\
& +\sum_{l=0}^{D-1} c_{l}(a(\epsilon t, \epsilon), z, \epsilon)\left(\mathcal{D}_{\epsilon, \alpha}\left(\partial_{t}\right)\right)^{\delta_{l}} R_{l}\left(\partial_{z}\right) u(t, z, \epsilon)+f(a(\epsilon t, \epsilon), z, \epsilon)
\end{aligned}
$$

for given vanishing initial data $u(0, z, \epsilon) \equiv 0$. The precise shape of the function $a(\epsilon t, \epsilon)$ is displayed later in the work at (21) and will be justified by the approach we undertake in this work.

The coefficients $c_{l}$ and the forcing term $f$ are described as follows. For each $0 \leq l \leq D-1$, we consider a sequence of functions $m \mapsto C_{l, n}(m, \epsilon)$, for $n \geq 0$, i.e., part of the Banach space $E_{(\beta, \mu)}$ for some parameter $\beta, \mu>0$ (see Definition 1) and depends in an analytic way on $\epsilon$ on a disc $D\left(0, \epsilon_{0}\right)$ and for which two constants $C_{l, 1}>0, C_{l, 2}>0$ can be found with

$$
\sup _{\epsilon \in D\left(0, \epsilon_{0}\right)}\left\|C_{l, n}(m, \epsilon)\right\|_{(\beta, \mu)} \leq C_{l, 1}\left(C_{l, 2}\right)^{n}
$$

for any $n \geq 0$. We set the sequence of functions $c_{l, n}(z, \epsilon)$ defined as the Fourier transform of $C_{l, n}(m, \epsilon)$,

$$
c_{l, n}(z, \epsilon)=\frac{1}{(2 \pi)^{1 / 2}} \int_{-\infty}^{+\infty} C_{l, n}(m, \epsilon) e^{i z m} d m
$$

whenever $0 \leq l \leq D-1$ and $n \geq 0$. We define the coefficient $c_{l}$ as the function

$$
c_{l}(a(\epsilon t, \epsilon), z, \epsilon)=c_{l, 0}(z, \epsilon)+\sum_{n \geq 0} c_{l, n}(z, \epsilon)(a(\epsilon t, \epsilon))^{-n}
$$

We consider a family of functions $m \mapsto \psi_{n}(m, \epsilon)$, for $n \geq 1$ that belong to the Banach space $E_{(\beta, \mu)}$ and rely analytically on $\epsilon$ in the disc $D\left(0, \epsilon_{0}\right)$ and for which constants $D_{1}>0, D_{2}>0$ can be singled out with

$$
\sup _{\epsilon \in D\left(0, \epsilon_{0}\right)}\left\|\psi_{n}(m, \epsilon)\right\|_{(\beta, \mu)} \leq D_{1}\left(D_{2}\right)^{n}
$$

for all $n \geq 1$. We set the functions $d_{n}(z, \epsilon)$ as the Fourier transform of $\psi_{n}(m, \epsilon)$,

$$
d_{n}(z, \epsilon)=\frac{1}{(2 \pi)^{1 / 2}} \int_{-\infty}^{+\infty} \psi_{n}(m, \epsilon) e^{i z m} d m
$$

provided that $n \geq 1$. We define the forcing term $f(a(\epsilon t, \epsilon), z, \epsilon)$ as the sum

$$
f(a(\epsilon t, \epsilon), z, \epsilon)=\sum_{n \geq 1} d_{n}(z, \epsilon)(a(\epsilon t, \epsilon))^{-n}
$$

The domains where these functions (14) and (17) are well defined and holomorphic will be specified later in the paper in Theorems 1 and 2.

Throughout this work, we are searching for time rescaled solutions of (11) with the shape

$$
u(t, z, \epsilon)=U(\epsilon t, z, \epsilon)
$$

In fact, if we define the next differential operator

$$
\mathcal{D}_{\epsilon, \alpha}\left(\partial_{T}\right)=\left(T^{2}-\epsilon^{\alpha+1}\right) \partial_{T}
$$


the expression $U(T, z, \epsilon)$, by means of the change of variable $T=\epsilon t$, is required to solve the next singular problem

$$
\begin{aligned}
Q\left(\partial_{z}\right) U(T, z, \epsilon)=c_{1,2} Q_{1}\left(\partial_{z}\right) & U(T, z, \epsilon) Q_{2}\left(\partial_{z}\right) U(T, z, \epsilon)+\left(\mathcal{D}_{\epsilon, \alpha}\left(\partial_{T}\right)\right)^{\delta_{D}} R_{D}\left(\partial_{z}\right) U(T, z, \epsilon) \\
& +\sum_{l=0}^{D-1} c_{l}(a(T, \epsilon), z, \epsilon)\left(\mathcal{D}_{\epsilon, \alpha}\left(\partial_{T}\right)\right)^{\delta_{l}} R_{l}\left(\partial_{z}\right) U(T, z, \epsilon)+f(a(T, \epsilon), z, \epsilon)
\end{aligned}
$$

We need to set forth the definition of Banach spaces that have already been introduced in [1].

Definition 3. Let $S_{d}$ be an unbounded sector centered at 0 with bisecting direction $d \in \mathbb{R}$ and $D(0, \rho)$ the open disc centered at 0 with radius $\rho>0$. We fix $v, \beta>0$ and $\mu>1$ some positive real numbers. We set $E_{(\nu, \beta, \mu)}^{d}$ as the vector space of continuous functions $(\tau, m) \mapsto h(\tau, m)$ on $\left(S_{d} \cup D(0, \rho)\right) \times \mathbb{R}$, holomorphic relatively to $\tau$ on $S_{d} \cup D(0, \rho)$ for which the norm

$$
\|h(\tau, m)\|_{(\nu, \beta, \mu)}=\sup _{\tau \in S_{d} \cup D(0, \rho), m \in \mathbb{R}}(1+|m|)^{\mu} e^{\beta|m|} \frac{1+|\tau|^{2}}{|\tau|} \exp (-v|\tau|)|h(\tau, m)|
$$

is finite. The space $E_{(\nu, \beta, \mu)}^{d}$ equipped with the norm $\|\cdot\|_{(\nu, \beta, \mu)}$ is a Banach space.

At a first stage, we undertake a similar strategy as in $[7,8]$ and seek for solutions to $(18)$ in the form of special Laplace and Fourier transforms

$$
U_{\gamma}(T, z, \epsilon)=\frac{1}{(2 \pi)^{1 / 2}} \int_{-\infty}^{+\infty} \int_{L_{\gamma}} W(\tau, m, \epsilon) \exp (\tau a(T, \epsilon)) e^{i z m} \frac{d \tau}{\tau} d m
$$

where $L_{\gamma}=\mathbb{R}_{+} e^{\sqrt{-1} \gamma}$ stands for a halfline with direction $\gamma \in \mathbb{R}$, with $L_{\gamma} \subset S_{d} \cup\{0\}$, where $S_{d}$ represents a sector as described in Definition 3 and $a(T, \epsilon)$ stands for a well selected analytic function. Within this section, we assume that the map $(\tau, m) \mapsto W(\tau, m, \epsilon)$ belongs to the Banach space $E_{(v, \beta, \mu)}^{d}$ given in Definition 3 provided that $\epsilon \in D\left(0, \epsilon_{0}\right)$.

\subsection{Statement of a Convolution Problem Satisfied by the Expression $W(\tau, m, \epsilon)$}

Our principal intention is to find a related tractable problem fulfilled by the function $W$. In this respect, the function $a(T, \epsilon)$ is properly singled out in a way that the next identity formally holds

$$
\mathcal{D}_{\epsilon, \alpha}\left(\partial_{T}\right) U_{\gamma}(T, z, \epsilon)=\frac{1}{(2 \pi)^{1 / 2}} \int_{-\infty}^{+\infty} \int_{L_{\gamma}} \tau W(\tau, m, \epsilon) \exp (\tau a(T, \epsilon)) e^{i z m} \frac{d \tau}{\tau} d m
$$

Specifically, the function $a(T, \epsilon)$ can be chosen as any primitive of the rational function $1 /\left(s^{2}-\right.$ $\left.\epsilon^{\alpha+1}\right)$. Among them, we pick up the simplest one

$$
a(T, \epsilon)=\frac{1}{2 \epsilon^{\frac{\alpha+1}{2}}} \log \left(\frac{T-\epsilon^{\frac{\alpha+1}{2}}}{T+\epsilon^{\frac{\alpha+1}{2}}}\right)
$$

For later need, the next lemma is also easily checked. The proof follows from the application of Definition 2 (b) and the use of Fubini theorem.

Lemma 1. (1) Let $(\tau, m) \mapsto H_{1}(\tau, m, \epsilon)$ be a function that belongs to $E_{(\nu, \beta, \mu)}^{d}$ whenever $\epsilon \in D\left(0, \epsilon_{0}\right)$. We set

$$
V_{1, \gamma}(T, z, \epsilon)=\frac{1}{(2 \pi)^{1 / 2}} \int_{-\infty}^{+\infty} \int_{L_{\gamma}} H_{1}(\tau, m, \epsilon) \exp (\tau a(T, \epsilon)) e^{i z m} \frac{d \tau}{\tau} d m
$$


Then, the next identity holds

$$
\begin{aligned}
& V_{1, \gamma}(T, z, \epsilon) U_{\gamma}(T, z, \epsilon) \\
& =\frac{1}{(2 \pi)^{1 / 2}} \int_{-\infty}^{+\infty} \int_{L_{\gamma}} H_{1}(\tau, m, \epsilon)_{*_{\tau}}^{*_{m}} W(\tau, m, \epsilon) \exp (\tau a(T, \epsilon)) e^{i z m} \frac{d \tau}{\tau} d m
\end{aligned}
$$

where

$$
H_{1}(\tau, m, \epsilon)_{*_{\tau}}^{*_{m}} W(\tau, m, \epsilon):=\frac{1}{(2 \pi)^{1 / 2}} \int_{-\infty}^{+\infty} \tau \int_{0}^{\tau} H_{1}\left(\tau-s, m-m_{1}, \epsilon\right) W\left(s, m_{1}, \epsilon\right) \frac{d s}{(\tau-s) s} d m_{1}
$$

(2) Let $m \mapsto H_{2}(m, \epsilon)$ be an element of $E_{(\beta, \mu)}$ for any given $\epsilon \in D\left(0, \epsilon_{0}\right)$. We put

$$
V_{2}(z, \epsilon)=\frac{1}{(2 \pi)^{1 / 2}} \int_{-\infty}^{+\infty} H_{2}(m, \epsilon) e^{i z m} d m
$$

The following identity

$$
V_{2}(z, \epsilon) U_{\gamma}(T, z, \epsilon)=\frac{1}{(2 \pi)^{1 / 2}} \int_{-\infty}^{+\infty} \int_{L_{\gamma}} H_{2}(m, \epsilon) *_{m} W(\tau, m, \epsilon) \exp (\tau a(T, \epsilon)) e^{i z m} \frac{d \tau}{\tau} d m
$$

holds with

$$
H_{2}(m, \epsilon) *_{m} W(\tau, m, \epsilon)=\frac{1}{(2 \pi)^{1 / 2}} \int_{-\infty}^{+\infty} H_{2}\left(m-m_{1}, \epsilon\right) W\left(\tau, m_{1}, \epsilon\right) d m_{1}
$$

Moreover, we observe that for any natural number $h \geq 1$, the building block $(a(T, \epsilon))^{-h}$ can be easily expressed as special Laplace transforms that involve the function $a(T, \epsilon)$ described in (21). Indeed, a direct computation shows that

$$
(a(T, \epsilon))^{-h}=\frac{(-1)^{h}}{\Gamma(h)} \int_{L_{\gamma}} \tau^{h} \exp (\tau a(T, \epsilon)) \frac{d \tau}{\tau}
$$

for any integer $h \geq 1$ provided that the integral makes sense for a properly chosen path $L_{\gamma}$. As a result, we can express the coefficients $c_{l}$, for $0 \leq l \leq D-1$ and forcing term $f$ as special Laplace and Fourier transforms. Specifically, according to the integral representations (13) and (16) and the expansions (14) and (17), we obtain

$$
\begin{aligned}
c_{l}(a(T, \epsilon), z, \epsilon)= & \frac{1}{(2 \pi)^{1 / 2}} \int_{-\infty}^{+\infty} C_{l, 0}(m, \epsilon) e^{i z m} d m \\
& +\frac{1}{(2 \pi)^{1 / 2}} \int_{-\infty}^{+\infty} \int_{L_{\gamma}} C_{l}(\tau, m, \epsilon) \exp (\tau a(T, \epsilon)) e^{i z m} \frac{d \tau}{\tau} d m
\end{aligned}
$$

where

$$
C_{l}(\tau, m, \epsilon)=\sum_{n \geq 1} C_{l, n}(m, \epsilon) \frac{(-\tau)^{n}}{\Gamma(n)}
$$

and

$$
f(a(T, \epsilon), z, \epsilon)=\frac{1}{(2 \pi)^{1 / 2}} \int_{-\infty}^{+\infty} \int_{L_{\gamma}} \psi(\tau, m, \epsilon) \exp (\tau a(T, \epsilon)) e^{i z m} \frac{d \tau}{\tau} d m
$$

where

$$
\psi(\tau, m, \epsilon)=\sum_{n \geq 1} \psi_{n}(m, \epsilon) \frac{(-\tau)^{n}}{\Gamma(n)}
$$


We are now ready to state the main convolution problem satisfied by the map $(\tau, m) \mapsto W(\tau, m, \epsilon)$ provided that $U_{\gamma}$ given by (19) solves (18). Specifically,

$$
\begin{aligned}
& Q(i m) W(\tau, m, \epsilon)=c_{1,2} Q_{1}(i m) W(\tau, m, \epsilon)_{* \tau}^{*_{m}} Q_{2}(i m) W(\tau, m, \epsilon)+\tau^{\delta_{D}} R_{D}(i m) W(\tau, m, \epsilon) \\
& +\sum_{l=0}^{D-1}\left\{\left(C_{l, 0}(m, \epsilon) *_{m} \tau^{\delta_{l}} R_{l}(i m) W(\tau, m, \epsilon)\right)+\left(C_{l}(\tau, m, \epsilon)_{* \tau}^{*_{m}} \tau^{\delta_{l}} R_{l}(i m) W(\tau, m, \epsilon)\right)\right\} \\
& +\psi(\tau, m, \epsilon)
\end{aligned}
$$

\section{Resolution of the Convolution Problem in Analytic and Continuous Function Spaces}

\subsection{Bounds for Convolution Operators Acting on Banach Spaces with Exponential Growth and Decay}

We keep the notations of the previous sections and subsections. Throughout this subsection, we establish a list of technical lemmas that are essential in the resolution of the problem (29) within the Banach spaces presented in Definition 3 from Section 2.2. Although the proofs of the statements are close to the ones given in [1], they will be given in full details for the sake of clarity and readability of the paper.

In a first lemma, we observe that the functions $C_{l}$ and $\psi$ defined in (26) and (28) belong to the Banach space $E_{(v, \beta, \mu)}^{d}$.

Lemma 2. For all $0 \leq l \leq D-1$, all $\epsilon \in D\left(0, \epsilon_{0}\right)$, the functions $(\tau, m) \mapsto C_{l}(\tau, m, \epsilon)$ and $(\tau, m) \mapsto$ $\psi(\tau, m, \epsilon)$ belong to the Banach spaces $E_{(\nu, \beta, \mu)}^{d}$ for any unbounded sector $S_{d}$, any disc $D(0, \rho)$, for the parameters $\beta, \mu$ fixed in Section 2.2 and for any $v>\max \left(C_{l, 2}, D_{2}\right)$ for all $0 \leq l \leq D-1$. Moreover, there exist constants $C_{l, 3}>0$ (relying on $C_{l, 2}$ and $v$ ) with

$$
\sup _{\epsilon \in D\left(0, \epsilon_{0}\right)}\left\|C_{l}(\tau, m, \epsilon)\right\|_{(\nu, \beta, \mu)} \leq C_{l, 1} C_{l, 3}
$$

for all $0 \leq l \leq D-1$ and one can select a constant $D_{3}>0$ (which depends on $D_{2}$ and $v$ ) for which

$$
\sup _{\epsilon \in D\left(0, \epsilon_{0}\right)}\|\psi(\tau, m, \epsilon)\|_{(\nu, \beta, \mu)} \leq D_{1} D_{3}
$$

holds.

Proof. According to the assumption (12), for any given $\tilde{C}_{l, 2}>C_{l, 2}$, one can find a constant $C_{l, 3}>0$ such that

$$
\begin{array}{r}
\left|C_{l}(\tau, m, \epsilon)\right| \leq C_{l, 1} e^{-\beta|m|}(1+|m|)^{-\mu} \sum_{n \geq 1} \frac{\left(C_{l, 2}|\tau|\right)^{n}}{(n-1) !}=C_{l, 1} C_{l, 2} e^{-\beta|m|}(1+|m|)^{-\mu}|\tau| \exp \left(C_{l, 2}|\tau|\right) \\
\leq C_{l, 1} C_{l, 2} C_{l, 3} e^{-\beta|m|}(1+|m|)^{-\mu} \frac{|\tau|}{1+|\tau|^{2}} \exp \left(\tilde{C}_{l, 2}|\tau|\right)
\end{array}
$$

for all $\tau \in S_{d} \cup D(0, \rho)$, all $m \in \mathbb{R}$ and all $\epsilon \in D\left(0, \epsilon_{0}\right)$. This yields the first bounds (30). Similar estimates can be obtained for $\psi(\tau, m, \epsilon)$ owing to the conditions (15) which give rise to the second bounds (31).

In the next lemma, we check the continuity property for the operator of multiplication by a bounded function. Its proof is straightforward. 
Lemma 3. Let $(\tau, m) \mapsto a(\tau, m)$ be a bounded continuous function on $\left(S_{d} \cup D(0, \rho)\right) \times \mathbb{R}$, i.e., holomorphic regarding $\tau$ on $S_{d} \cup D(0, \rho)$. Then,

$$
\|a(\tau, m) h(\tau, m)\|_{(\nu, \beta, \mu)} \leq\left(\sup _{\tau \in S_{d} \cup D(0, \rho), m \in \mathbb{R}}|a(\tau, m)|\right)\|h(\tau, m)\|_{(\nu, \beta, \mu)}
$$

whenever $h(\tau, m) \in E_{(\nu, \beta, \mu)}^{d}$.

In the sequel, we need to fix some holomorphic function $a_{\delta_{D}}(\tau)$ on $S_{d} \cup D(0, \rho)$, continuous on its closure $\bar{S}_{d} \cup \bar{D}(0, \rho)$, with the bounds

$$
\frac{1}{(1+|\tau|)^{\delta_{D}}} \leq\left|a_{\delta_{D}}(\tau)\right| \leq \frac{2}{(1+|\tau|)^{\delta_{D}}}
$$

for all $\tau \in \bar{S}_{d} \cup \bar{D}(0, \rho)$.

In the next three lemmas, we analyze the continuity of convolutions operators acting on $E_{(\nu, \beta, \mu)}^{d}$.

Lemma 4. Assume the condition

$$
\mu>\max \left(\operatorname{deg}\left(Q_{1}\right)+1, \operatorname{deg}\left(Q_{2}\right)+1\right)
$$

holds. Then, we can find a constant $K_{1}>0$ (which rests on $Q_{1}, Q_{2}, R_{D}, \mu$ ) with

$$
\begin{gathered}
\left\|\frac{1}{R_{D}(i m)} \times\left\{Q_{1}(i m) h(\tau, m)_{*_{\tau}}^{*_{m}} Q_{2}(i m) g(\tau, m)\right\}\right\|_{(\nu, \beta, \mu)} \\
\leq K_{1}\|h(\tau, m)\|_{(\nu, \beta, \mu)}\|g(\tau, m)\|_{(\nu, \beta, \mu)}
\end{gathered}
$$

for all $h(\tau, m), g(\tau, m) \in E_{(\nu, \beta, \mu)}^{d}$.

Proof. Owing to Definition 3, the function $h(\tau, m)$ suffers the bounds

$$
|h(\tau, m)| \leq\|h(\tau, m)\|_{(\nu, \beta, \mu)}(1+|m|)^{-\mu} e^{-\beta|m|} \frac{|\tau|}{1+|\tau|^{2}} \exp (\nu|\tau|)
$$

for all $\tau \in S_{d} \cup D(0, \rho)$, all $m \in \mathbb{R}$. Correspondingly, the function $g(\tau, m)$ is submitted to similar estimates. We introduce a constant $K>0$ defined by

$$
K=\sup _{x \geq 0}\left(1+x^{2}\right) \int_{0}^{x} \frac{1}{\left(1+(x-h)^{2}\right)\left(1+h^{2}\right)} d h
$$

that has been computed in Corollary 4.9 of [28]. Then, the next estimates hold

$$
\begin{aligned}
\mid \frac{1}{R_{D}(i m)(2 \pi)^{1 / 2}} \int_{-\infty}^{+\infty} \tau \int_{0}^{\tau} Q_{1}\left(i\left(m-m_{1}\right)\right) h\left(\tau-s, m-m_{1}\right) \\
\times\left. Q_{2}\left(i m_{1}\right) g\left(s, m_{1}\right) \frac{d s}{(\tau-s) s} d m_{1}\left|\leq \frac{1}{(2 \pi)^{1 / 2}}\right||h(\tau, m)|\right|_{(v, \beta, \mu)}|| g(\tau, m)||_{(v, \beta, \mu)} \\
\quad \times|\tau| \int_{0}^{|\tau|} \frac{1}{\left(1+(|\tau|-h)^{2}\right)\left(1+h^{2}\right)} \exp (v(|\tau|-h)) \exp (v h) d h \\
\quad \times \frac{1}{\left|R_{D}(i m)\right|} \int_{-\infty}^{+\infty} \frac{\left|Q_{1}\left(i\left(m-m_{1}\right)\right)\right|\left|Q_{2}\left(i m_{1}\right)\right|}{\left(1+\left|m-m_{1}\right|\right)^{\mu}\left(1+\left|m_{1}\right|\right)^{\mu}} e^{-\beta\left|m-m_{1}\right|} e^{-\beta\left|m_{1}\right|} d m_{1} \\
\leq \frac{K}{(2 \pi)^{1 / 2}}|| h(\tau, m)||_{(\nu, \beta, \mu)}|| g(\tau, m)||_{(\nu, \beta, \mu)} \mathcal{A}(m) \frac{|\tau|}{1+|\tau|^{2}} \exp (v|\tau|)
\end{aligned}
$$


where

$$
\mathcal{A}(m)=\frac{1}{\left|R_{D}(i m)\right|} \int_{-\infty}^{+\infty} \frac{\left|Q_{1}\left(i\left(m-m_{1}\right)\right)\right|\left|Q_{2}\left(i m_{1}\right)\right|}{\left(1+\left|m-m_{1}\right|\right)^{\mu}\left(1+\left|m_{1}\right|\right)^{\mu}} e^{-\beta\left|m-m_{1}\right|} e^{-\beta\left|m_{1}\right|} d m_{1}
$$

Since $Q_{1}, Q_{2}, R_{D}$ are polynomials withstanding (10), we can find constants $\mathfrak{Q}_{1}, \mathfrak{Q}_{2}, \mathfrak{R}_{D}>0$ with

$$
\left|Q_{j}\left(i\left(m-m_{1}\right)\right)\right| \leq \mathfrak{Q}_{j}\left(1+\left|m-m_{1}\right|\right)^{\operatorname{deg}\left(Q_{j}\right)}, \quad\left|R_{D}(i m)\right| \geq \mathfrak{R}_{D}(1+|m|)^{\operatorname{deg}\left(R_{D}\right)}
$$

for $j=1,2$, all $m, m_{1} \in \mathbb{R}$. Bearing in mind the triangular inequality $|m| \leq\left|m_{1}\right|+\left|m-m_{1}\right|$ whenever $m, m_{1} \in \mathbb{R}$, we deduce that

$$
\mathcal{A}(m) \leq(1+|m|)^{-\mu} e^{-\beta|m|} \mathcal{A}_{1}
$$

for

$$
\mathcal{A}_{1}=\frac{\mathfrak{Q}_{1} \mathfrak{Q}_{2}}{\mathfrak{R}_{D}} \sup _{m \in \mathbb{R}}(1+|m|)^{\mu-\operatorname{deg}\left(R_{D}\right)} \int_{-\infty}^{+\infty} \frac{1}{\left(1+\left|m-m_{1}\right|\right)^{\mu-\operatorname{deg}\left(Q_{1}\right)}\left(1+\left|m_{1}\right|\right)^{\mu-\operatorname{deg}\left(Q_{2}\right)}} d m_{1}
$$

that turns out to be a finite positive number under the two conditions imposed above (10) and (34) according to Lemma 4 of [29] or Lemma 2.2 of [30]. At last, gathering (38) and (40) yields the expected bounds (35).

Lemma 5. Take for granted that

$$
\mu>\operatorname{deg}\left(R_{l}\right)+1
$$

for all $0 \leq l \leq D-1$ holds. Then, there exists a constant $K_{2}>0$ (relying on $\mu, \delta_{l}, R_{l}$ for $0 \leq l \leq D$ ) with

$$
\begin{gathered}
\left\|\frac{a_{\delta_{D}}(\tau)}{R_{D}(i m)} \times\left\{C_{l, 0}(m, \epsilon) *_{m} \tau^{\delta_{l}} R_{l}(i m) h(\tau, m)\right\}\right\|_{(\nu, \beta, \mu)} \\
\leq K_{2}\left\|C_{l, 0}(m, \epsilon)\right\|_{(\beta, \mu)}\|h(\tau, m)\|_{(\nu, \beta, \mu)}
\end{gathered}
$$

for all $h(\tau, m) \in E_{(\nu, \beta, \mu)^{\prime}}^{d}$ all $\epsilon \in D\left(0, \epsilon_{0}\right)$.

Proof. By definition, the function $h(\tau, m)$ is submitted to the above bounds (36). By construction, $C_{l, 0}(m, \epsilon)$ satisfies

$$
\left|C_{l, 0}(m, \epsilon)\right| \leq\left\|C_{l, 0}(m, \epsilon)\right\|_{(\beta, \mu)}(1+|m|)^{-\mu} e^{-\beta|m|}
$$

for all $m \in \mathbb{R}, \epsilon \in D\left(0, \epsilon_{0}\right)$. We deduce the following upper bounds

$$
\begin{array}{r}
\left|\frac{a_{\delta_{D}}(\tau)}{(2 \pi)^{1 / 2} R_{D}(i m)} \int_{-\infty}^{+\infty} C_{l, 0}\left(m-m_{1}, \epsilon\right) \tau^{\delta_{l}} R_{l}\left(i m_{1}\right) h\left(\tau, m_{1}\right) d m_{1}\right| \leq \frac{1}{(2 \pi)^{1 / 2}} \sup _{x \geq 0} \frac{2 x^{\delta_{l}}}{(1+x)^{\delta_{D}}} \\
\times\left\|C_{l, 0}(m, \epsilon)\right\|_{(\beta, \mu)}|| h(\tau, m) \|_{(\nu, \beta, \mu)} \mathcal{B}(m) \frac{|\tau|}{1+|\tau|^{2}} \exp (v|\tau|)
\end{array}
$$

where

$$
\mathcal{B}(m)=\frac{1}{\left|R_{D}(i m)\right|} \int_{-\infty}^{+\infty} \frac{1}{\left(1+\left|m-m_{1}\right|\right)^{\mu}} \frac{\left|R_{l}\left(i m_{1}\right)\right|}{\left(1+\left|m_{1}\right|\right)^{\mu}} e^{-\beta\left|m-m_{1}\right|} e^{-\beta\left|m_{1}\right|} d m_{1}
$$

Since $R_{l}$ are polynomials under the restriction (10), some constants $\Re_{l}>0$ can be found such that

$$
\left|R_{l}(i m)\right| \leq \mathfrak{R}_{l}(1+|m|)^{\operatorname{deg}\left(R_{l}\right)}
$$

for $0 \leq l \leq D-1$. Owing to the triangular inequality and the lower bounds for $R_{D}$ in (39), we get that

$$
\mathcal{B}(m) \leq(1+|m|)^{-\mu} e^{-\beta|m|} \mathcal{B}_{1}
$$


for

$$
\mathcal{B}_{1}=\frac{\mathfrak{R}_{l}}{\mathfrak{R}_{D}} \sup _{m \in \mathbb{R}}(1+|m|)^{\mu-\operatorname{deg}\left(R_{D}\right)} \int_{-\infty}^{+\infty} \frac{1}{\left(1+\left|m-m_{1}\right|\right)^{\mu}\left(1+\left|m_{1}\right|\right)^{\mu-\operatorname{deg}\left(R_{l}\right)}} d m_{1}
$$

a finite positive real number under the conditions (10) and (41) in accordance with Lemma 4 of [29] or Lemma 2.2 of [30]. The lemma finally follows from (43) with (44).

Lemma 6. Under the condition (41), we can select a constant $K_{3}>0$ (hinging on $\mu, \delta_{l}, R_{l}$ for $0 \leq l \leq D$ ) such that

$$
\begin{gathered}
\left\|\frac{a_{\delta_{D}}(\tau)}{R_{D}(i m)} \times\left\{C_{l}(\tau, m, \epsilon)_{* \tau}^{* m} \tau^{\delta_{l}} R_{l}(i m) h(\tau, m)\right\}\right\|_{(\nu, \beta, \mu)} \\
\leq K_{3}\left\|C_{l}(\tau, m, \epsilon)\right\|_{(\nu, \beta, \mu)}\|h(\tau, m)\|_{(\nu, \beta, \mu)}
\end{gathered}
$$

for all $h(\tau, m) \in E_{(v, \beta, \mu)}^{d}$

Proof. From Lemma 2, we know that $C_{l}(\tau, m, \epsilon)$ belongs to $E_{(v, \beta, \mu)}^{d}$ and fulfills the bounds

$$
\left|C_{l}(\tau, m, \epsilon)\right| \leq \|\left. C_{l}(\tau, m, \epsilon)\right|_{(v, \beta, \mu)}(1+|m|)^{-\mu} e^{-\beta|m|} \frac{|\tau|}{1+|\tau|^{2}} \exp (v|\tau|)
$$

for all $\tau \in S_{d} \cup D(0, \rho)$, all $m \in \mathbb{R}$. Likewise, $h(\tau, m)$ is subjected to the bounds (36). Taking into account the definition of the constant $K$ in (37), the next estimates follow

$$
\begin{aligned}
& \mid \frac{a_{\delta_{D}}(\tau)}{R_{D}(i m)(2 \pi)^{1 / 2}} \int_{-\infty}^{+\infty} \tau \int_{0}^{\tau} C_{l}\left(\tau-s, m-m_{1}, \epsilon\right) s^{\delta_{l}} R_{l}\left(i m_{1}\right) h\left(s, m_{1}\right) \frac{d s}{(\tau-s) s} d m_{1} \mid \\
& \leq \frac{1}{(2 \pi)^{1 / 2}}|| C_{l}(\tau, m, \epsilon)||_{(v, \beta, \mu)}|| h(\tau, m)||_{(v, \beta, \mu)} \sup _{x \geq 0} \frac{2 x^{\delta_{l}}}{(1+x)^{\delta_{D}}} \\
& \times|\tau| \int_{0}^{|\tau|} \frac{1}{\left(1+(|\tau|-h)^{2}\right)\left(1+h^{2}\right)} \exp (v(|\tau|-h)) \exp (v h) d h \\
& \quad \times \frac{1}{\left|R_{D}(i m)\right|} \int_{-\infty}^{+\infty} \frac{\left|R_{l}\left(i m_{1}\right)\right|}{\left(1+\left|m-m_{1}\right|\right)^{\mu}\left(1+\left|m_{1}\right|\right)^{\mu}} e^{-\beta\left|m-m_{1}\right|} e^{-\beta\left|m_{1}\right|} d m_{1} \\
& \leq\left.\left.\frac{K}{(2 \pi)^{1 / 2}} \sup _{x \geq 0} \frac{2 x^{\delta_{l}}}{(1+x)^{\delta_{D}}}|| C_{l}(\tau, m, \epsilon)\right|_{(v, \beta, \mu)}|| h(\tau, m)\right|_{(\nu, \beta, \mu)} \mathcal{B}(m) \frac{|\tau|}{1+|\tau|^{2}} \exp (v|\tau|)
\end{aligned}
$$

where $\mathcal{B}(m)$ is given in the proof of Lemma 5 and satisfies the bounds (44) under the constraints (10) and (41). Lemma 6 follows.

\subsection{Construction of a Unique Solution to the Convolution Equation}

The principal objective of this subsection consists of the construction of a unique solution of the equation (29) within the Banach space described in Definition 3.

We first provide further analytic assumptions on the leading polynomials $Q(X)$ and $R_{D}(X)$ that allows us to transform our problem (29) into a fixed-point equation which is stated later on, see (64). Specifically, we impose the existence of an unbounded sectorial annulus

$$
A_{Q, R_{D}}=\left\{z \in \mathbb{C} /|z| \geq r_{Q, R_{D}} \arg (z) \in\left(\alpha_{Q, R_{D}}, \beta_{Q, R_{D}}\right)\right\}
$$

for some inner radius $r_{Q, R_{D}}>0$ and angles $\alpha_{Q, R_{D}}, \beta_{Q, R_{D}} \in \mathbb{R}$ with $\alpha_{Q, R_{D}}<\beta_{Q, R_{D}}$ such that

$$
\frac{Q(i m)}{R_{D}(i m)} \in A_{Q, R_{D}}
$$


for all $m \in \mathbb{R}$. At this point, we follow a comparable protocol as the one initiated in our erstwhile study [1]. We focus on the polynomial $P_{m}(\tau)=Q(i m)-\tau^{\delta_{D}} R_{D}(i m)$ for which we ask lower bounds respectively to $\tau$ in $\mathbb{C}$ and $m$ in $\mathbb{R}$. We factorize this polynomial as follows

$$
P_{m}(\tau)=-R_{D}(i m) \prod_{l=0}^{\delta_{D}-1}\left(\tau-q_{l}(m)\right)
$$

where the roots can be computed explicitly as

$$
q_{l}(m)=\left(\frac{|Q(i m)|}{\left|R_{D}(i m)\right|}\right)^{1 / \delta_{D}} \times \exp \left(\frac{\sqrt{-1}}{\delta_{D}}\left(\arg \left(\frac{Q(i m)}{R_{D}(i m)}\right)+2 \pi l\right)\right)
$$

We consider an unbounded sector $S_{d}$ centered at 0 with bisecting direction $d \in \mathbb{R}$ and a small disc $D(0, \rho)$. We adjust the annulus $A_{Q, R_{D}}$ in a way that the next condition hold: a constant $\mathfrak{q}>0$ can be chosen with lower bounds

$$
\left|\tau-q_{l}(m)\right| \geq \mathfrak{q}(1+|\tau|)
$$

for all $0 \leq l \leq \delta_{D}-1$, all $m \in \mathbb{R}$, whenever $\tau \in S_{d} \cup D(0, \rho)$. Indeed, the feature (47) impose all the roots $q_{l}(m), 0 \leq l \leq \delta_{D}-1$ to remain a part of the origin and satisfy $\left|q_{l}(m)\right|>2 \rho$ for a well-chosen $\rho>0$. Furthermore, provided that the aperture of $A_{Q, R_{D}}$ is taken close enough to 0 , all the roots $q_{l}(m)$ stay inside a union $\mathcal{U}$ of unbounded sectors centered at 0 that do not cover a full neighborhood of 0 in $\mathbb{C}^{*}$. We select the sector $S_{d}$ such that $S_{d} \cap \mathcal{U}=\varnothing$ holds. As a result, the quotient $q_{l}(m) / \tau$ is kept at a distance of some disc centered at 1 in $\mathbb{C}$ whenever $\tau \in S_{d}, m \in \mathbb{R}$ and $0 \leq l \leq \delta_{D}-1$. Then, Ref. (49) follows.

Lower bounds for $P_{m}(\tau)$ are given in the next lemma.

Lemma 7. When the sector $S_{d}$ and the disc $D(0, \rho)$ are submitted to the constraints stated above, a constant $C_{P}>0$ (relying on $\delta_{D}$ and $\mathfrak{q}$ ) can be singled out with

$$
\left|P_{m}(\tau)\right| \geq C_{P}\left|R_{D}(i m)\right|(1+|\tau|)^{\delta_{D}}
$$

for all $\tau \in S_{d} \cup D(0, \rho)$, all $m \in \mathbb{R}$.

Proof. The factorization (48) together with (49) lead to

$$
\left|P_{m}(\tau)\right| \geq \mathfrak{q}^{\delta_{D}}\left|R_{D}(i m)\right|(1+|\tau|)^{\delta_{D}}
$$

provided that $\tau \in S_{d} \cup D(0, \rho)$ and $m \in \mathbb{R}$.

In the next lemma, we discuss properties of a nonlinear map $\mathcal{H}_{\epsilon}$ which acts on a small closed ball centered at the origin in the Banach space $E_{(\nu, \beta, \mu)}^{d}$.

Lemma 8. Assume that the sector $S_{d}$ and the disc $D(0, \rho)$ fulfill the constraints stated above. Under the additional conditions that

$$
\mu>\max \left(\operatorname{deg}\left(Q_{1}\right)+1, \operatorname{deg}\left(Q_{2}\right)+1\right), \quad \mu>\operatorname{deg}\left(R_{l}\right)+1
$$


for $0 \leq l \leq D-1$ and provided that the constants $c_{1,2}, C_{l, 1}, D_{1}$ for $0 \leq l \leq D-1$, defined in Section 2.2 are taken small enough, for all $\epsilon \in D\left(0, \epsilon_{0}\right)$, the map set as

$$
\begin{aligned}
\mathcal{H}_{\epsilon}(h(\tau, m)):=\frac{c_{1,2}}{P_{m}(\tau)} \times & \left\{Q_{1}(i m) h(\tau, m)_{*_{\tau}}^{*_{m}} Q_{2}(i m) h(\tau, m)\right\} \\
+ & \sum_{l=0}^{D-1}\left\{\frac{1}{P_{m}(\tau)} \times\left(C_{l, 0}(m, \epsilon) *_{m} \tau^{\delta_{l}} R_{l}(i m) h(\tau, m)\right)\right. \\
& \left.\quad+\frac{1}{P_{m}(\tau)} \times\left(C_{l}(\tau, m, \epsilon)_{*_{\tau}}^{*_{m}} \tau^{\delta_{l}} R_{l}(i m) h(\tau, m)\right)\right\}+\frac{\psi(\tau, m, \epsilon)}{P_{m}(\tau)}
\end{aligned}
$$

is submitted to the next properties:

(i) There exists a radius $\omega>0$ (which can be chosen independently of $\epsilon$ in $D\left(0, \epsilon_{0}\right)$ ) such that the next inclusions

$$
\mathcal{H}_{\epsilon}(\bar{B}(0, \omega)) \subset \bar{B}(0, \omega)
$$

hold, where $\bar{B}(0, \omega)$ stands for the closed ball of radius $\omega>0$ centered at 0 in the space $E_{(v, \beta, \mu)}^{d}$.

(ii) The map $\mathcal{H}_{\epsilon}$ is shrinking on $\bar{B}(0, \omega)$, namely

$$
\left\|\mathcal{H}_{\epsilon}\left(h_{1}\right)-\mathcal{H}_{\epsilon}\left(h_{2}\right)\right\|_{(\nu, \beta, \mu)} \leq \frac{1}{2}\left\|h_{1}-h_{2}\right\|_{(\nu, \beta, \mu)}
$$

is valid for all $h_{1}, h_{2}$ inside the ball $\bar{B}(0, \omega)$.

Proof. We concentrate on the first feature i). Let us take $h(\tau, m)$ in the space $E_{(v, \beta, \mu)}^{d}$. Keeping in mind Lemma 3,4 together with (50) and the first condition of (51), we find a constant $K_{1}>0$ (depending on $\left.Q_{1}, Q_{2}, R_{D}, \mu\right)$ with

$$
\begin{aligned}
& \left\|\frac{1}{P_{m}(\tau)} \times\left\{Q_{1}(i m) h(\tau, m)_{*_{\tau}}^{*_{m}} Q_{2}(i m) h(\tau, m)\right\}\right\|_{(\nu, \beta, \mu)} \\
& \quad=\left\|\frac{R_{D}(i m)}{P_{m}(\tau)} \times \frac{1}{R_{D}(i m)} \times\left\{Q_{1}(i m) h(\tau, m)_{*_{\tau}}^{*_{m}} Q_{2}(i m) h(\tau, m)\right\}\right\|_{(\nu, \beta, \mu)} \leq \frac{K_{1}}{C_{P}}\|h(\tau, m)\|_{(\nu, \beta, \mu)}^{2}
\end{aligned}
$$

According to Lemma 3,5 under the second requirement of (51) and assumption (12), we get a constant $K_{2}>0$ (relying on $\mu, \delta_{l}, R_{l}$ for $0 \leq l \leq D$ ) with

$$
\begin{aligned}
\| \frac{1}{P_{m}(\tau)} \times( & \left.C_{l, 0}(m, \epsilon) *_{m} \tau^{\delta_{l}} R_{l}(i m) h(\tau, m)\right) \|_{(v, \beta, \mu)} \\
=\| \frac{R_{D}(i m)}{P_{m}(\tau) a_{\delta_{D}}(\tau)} & \times \frac{a_{\delta_{D}}(\tau)}{R_{D}(i m)} \times\left(C_{l, 0}(m, \epsilon) *_{m} \tau^{\delta_{l}} R_{l}(i m) h(\tau, m)\right) \|_{(\nu, \beta, \mu)} \\
& \leq \frac{K_{2}}{C_{P}}\left\|C_{l, 0}(m, \epsilon)\right\|_{(\beta, \mu)}\|h(\tau, m)\|_{(\nu, \beta, \mu)} \leq \frac{K_{2}}{C_{P}} C_{l, 1}\|h(\tau, m)\|_{(v, \beta, \mu)}
\end{aligned}
$$

for all $0 \leq l \leq D-1$. Taking heed of Lemma 3,6 and (30) of Lemma 2, subjected to the second constraint of (51), a constant $K_{3}>0$ (relying on $\mu, \delta_{l}, R_{l}$ for $0 \leq l \leq D$ ) can be selected with the bounds

$$
\begin{aligned}
& \left\|\frac{1}{P_{m}(\tau)} \times\left(C_{l}(\tau, m, \epsilon)_{* \tau}^{*_{m}} \tau^{\delta_{l}} R_{l}(i m) h(\tau, m)\right)\right\|_{(\nu, \beta, \mu)} \\
& =\left\|\frac{R_{D}(i m)}{P_{m}(\tau) a_{\delta_{D}}(\tau)} \times \frac{a_{\delta_{D}}(\tau)}{R_{D}(i m)} \times\left(C_{l}(\tau, m, \epsilon)_{*_{\tau}}^{*_{m}} \tau^{\delta_{l}} R_{l}(i m) h(\tau, m)\right)\right\|_{(\nu, \beta, \mu)} \\
& \quad \leq \frac{K_{3}}{C_{P}}\left\|C_{l}(\tau, m, \epsilon)\right\|_{(\nu, \beta, \mu)}\|h(\tau, m)\|_{(\nu, \beta, \mu)} \leq \frac{K_{3}}{C_{P}} C_{l, 1} C_{l, 3}\|h(\tau, m)\|_{(\nu, \beta, \mu)}
\end{aligned}
$$


whenever $0 \leq l \leq D-1$. Furthermore, from (31) and (50), we notice that

$$
\left\|\frac{1}{P_{m}(\tau)} \psi(\tau, m, \epsilon)\right\|_{(\nu, \beta, \mu)} \leq \frac{1}{C_{P}} \sup _{m \in \mathbb{R}} \frac{1}{\left|R_{D}(i m)\right|} D_{1} D_{3}
$$

Now, we choose a positive real number $\mathcal{\omega}>0$ and select the constants $\left|c_{1,2}\right|, C_{l, 1}, D_{1}$ for $0 \leq l \leq$ $D-1$ close enough to 0 in a way that the next inequality holds:

$$
\left|c_{1,2}\right| \frac{K_{1}}{C_{P}} \mathfrak{\omega}^{2}+\sum_{l=0}^{D-1}\left\{\frac{K_{2} C_{l, 1}}{C_{P}} \mathfrak{\omega}+\frac{K_{3} C_{l, 1} C_{l, 3}}{C_{P}} \mathfrak{\omega}\right\}+\frac{1}{C_{P}} \sup _{m \in \mathbb{R}} \frac{1}{\left|R_{D}(i m)\right|} D_{1} D_{3} \leq \boldsymbol{\omega}
$$

As a result, provided that $h(\tau, m)$ is chosen such that $\|h(\tau, m)\|_{(\nu, \beta, \mu)} \leq \mathcal{W}$ and by collecting the list of bounds (55)-(58) under the constraint (59), the inclusion (53) holds.

In a second part of the proof, we discuss the second property ii). We pick up two elements $h_{1}(\tau, m)$ and $h_{2}(\tau, m)$ inside the ball $\bar{B}(0, \omega)$ constructed in the first part i).

We first deal with the nonlinear part of $\mathcal{H}_{\epsilon}$. To provide upper bounds, we need first to rewrite the next difference of convolutions into a sum of convolution terms that involve the difference $h_{1}-h_{2}$, namely

$$
\begin{aligned}
Q_{1}(i m) h_{1}(\tau, m)_{*_{\tau}}^{*_{m}} Q_{2}(i m) h_{1}(\tau, m)-Q_{1}(i m) & h_{2}(\tau, m)_{*_{\tau}}^{*_{m}} Q_{2}(i m) h_{2}(\tau, m) \\
=Q_{1}(i m) h_{1}(\tau, m){ }_{*_{\tau}}^{*_{m}}\left(Q_{2}(i m)\left(h_{1}(\tau, m)-h_{2}(\tau, m)\right)\right) & +\left(Q_{1}(i m)\left(h_{1}(\tau, m)-h_{2}(\tau, m)\right)\right)_{*_{\tau}^{*} Q_{2}(i m) h_{2}(\tau, m)}
\end{aligned}
$$

Then, under the first constraint of (51), Lemma 3, 4 with (50) ensure the existence of a constant $K_{1}>0$ (depending on $Q_{1}, Q_{2}, R_{D}$ and $\mu$ ) such that

$$
\begin{aligned}
\| \frac{1}{P_{m}(\tau)} \times\left\{Q_{1}(i m)\right. & h_{1}(\tau, m)_{*_{\tau}}^{*_{m}} Q_{2}(i m) h_{1}(\tau, m) \\
& \left.\quad-Q_{1}(i m) h_{2}(\tau, m)_{*_{\tau}}^{*_{m}} Q_{2}(i m) h_{2}(\tau, m)\right\} \|_{(v, \beta, \mu)} \\
= & \| \frac{R_{D}(i m)}{P_{m}(\tau)} \times \frac{1}{R_{D}(i m)} \times\left\{Q_{1}(i m) h_{1}(\tau, m)_{*_{\tau}}^{*_{m}} Q_{2}(i m) h_{1}(\tau, m)\right. \\
& \left.\quad-Q_{1}(i m) h_{2}(\tau, m)_{*_{\tau}}^{*_{m}} Q_{2}(i m) h_{2}(\tau, m)\right\} \|_{(v, \beta, \mu)} \\
\leq & \frac{K_{1}}{C_{P}}\left(\left\|h_{1}(\tau, m)\right\|_{(\nu, \beta, \mu)}+\left\|h_{2}(\tau, m)\right\|_{(\nu, \beta, \mu)}\right)\left\|h_{1}(\tau, m)-h_{2}(\tau, m)\right\|_{(v, \beta, \mu)}
\end{aligned}
$$

In this second phase, we sort the constants $\left|c_{1,2}\right|, C_{l, 1}$ for $0 \leq l \leq D-1$ small enough in order that the next inequality is fulfilled

$$
\left|c_{1,2}\right| \frac{K_{1}}{C_{P}} 2 \omega+\sum_{l=0}^{D-1}\left\{\frac{K_{2} C_{l, 1}}{C_{P}}+\frac{K_{3} C_{l, 1} C_{l, 3}}{C_{P}}\right\} \leq 1 / 2
$$

Then, gathering the bounds (61) and the corresponding estimates for the linear part of $\mathcal{H}_{\epsilon}$ through (56), (57) overhead where $h(\tau, m)$ has to be replaced by the difference $h_{1}(\tau, m)-h_{2}(\tau, m)$, we get the expected shrinking character of $\mathcal{H}_{\epsilon}$ given by (54).

Finally, we select the constants $\omega,\left|c_{1,2}\right|, C_{l, 1}, D_{1}$ for $0 \leq l \leq D-1$ in order that both restrictions (59) and (62) hold at the same time. This gives our lemma.

Now we reach the main result of this subsection.

Proposition 1. Take for granted that the hypotheses stated in Lemma 8 hold. Then, a proper choice of a real number $\omega>0$ and provided that the constants $\left|c_{1,2}\right|, C_{l, 1}, D_{1}$ for $0 \leq l \leq D-1$, are selected close enough to 0 , 
Equation (29) admits a unique solution $(\tau, m) \mapsto W(\tau, m, \epsilon)$ that belongs to the space $E_{(\nu, \beta, \mu)}^{d}$, for the sector $S_{d}$ and disc $D(0, \rho)$ chosen as above, with the boundedness property

$$
\|W(\tau, m, \epsilon)\|_{(v, \beta, \mu)} \leq \omega
$$

for every $\epsilon \in D\left(0, \epsilon_{0}\right)$.

Proof. From Lemma 8, we observe that the map $\mathcal{H}_{\epsilon}$ is contractive from the complete metric space $\bar{B}(0, \omega)$ into itself for the distance $d(x, y)=\|x-y\|_{(\nu, \beta, \mu)}$. As a result, the classical fixed-point theorem guarantees the existence of a unique function $(\tau, m) \mapsto W(\tau, m, \epsilon)$ inside $\bar{B}(0, \omega)$ with

$$
\mathcal{H}_{\epsilon}(W(\tau, m, \epsilon))=W(\tau, m, \epsilon)
$$

for every $\epsilon \in D\left(0, \epsilon_{0}\right)$. Moreover, this function $W$ relies analytically on $\epsilon$ in the disc $D\left(0, \epsilon_{0}\right)$.

Observe that if one puts the term $\tau^{\delta_{D}} R_{D}(i m) W(\tau, m, \epsilon)$ from the right to the left-hand side of Equation (29) and divide the resulting equation by the polynomial $P_{m}(\tau)$ given by (48), (29) can be exactly recast as the Equation (64) above. In conclusion, the unique fixed point of (64) precisely solves the problem (29) with vanishing initial data $W(0, m, \epsilon) \equiv 0$. The proposition follows.

\section{Construction of Analytic Solutions to the Main Problem and Their Parametric Asymptotic Expansions}

We build up two distinguished families of actual analytic solutions to our main problem (11). The elements of the first family are called outer solutions and those of the second family are named inner solutions in the context of so-called boundary layer expansions (see the textbook [31] for an explanation of this terminology). Indeed, the domain of holomorphy in time $t$ turns out to be a fixed sectorial annulus independent of $\epsilon$ that is kept apart of the origin for the outer family and a domain that relies on the parameter $\epsilon$ that comes close to the origin when $\epsilon$ tends to 0 for the inner family. Both sets of solutions lean on the next definition of so-called good covering in $\mathbb{C}^{*}$.

Definition 4. Let $\iota \geq 2$ be an integer. We consider a finite set $\underline{\mathcal{E}}=\left\{\mathcal{E}_{p}\right\}_{0 \leq p \leq l-1}$ where $\mathcal{E}_{p}$ stand for open sectors with vertex at 0 such that $\mathcal{E}_{p} \subset D\left(0, \epsilon_{0}\right)$ which fulfills the next three assumptions:

(i) $\mathcal{E}_{p} \cap \mathcal{E}_{p+1} \neq \varnothing$ for all $0 \leq p \leq \iota-1$ (with the convention that $\mathcal{E}_{\iota}=\mathcal{E}_{0}$ ).

(ii) $\mathcal{E}_{p_{1}} \cap \mathcal{E}_{p_{2}} \cap \mathcal{E}_{p_{3}}=\varnothing$ for any distinct integers $0 \leq p_{j} \leq \iota-1, j=1,2,3$.

(iii) The union of all the sectors $\mathcal{E}_{p}$ covers a punctured disc centered at 0 in $\mathbb{C}$.

Then, the set $\underline{\mathcal{E}}$ is called a good covering in $\mathbb{C}^{*}$.

\subsection{Outer Solutions}

We select a bounded sectorial annulus

$$
\mathcal{A}=\left\{t \in \mathbb{C} / \rho_{1}<|t|<\rho_{2}, \beta_{1}<\arg (t)<\beta_{2}\right\}
$$

for given radii $\rho_{1}, \rho_{2}>0$ and angles $\beta_{1}<\beta_{2}$.

The next lemma presents bounds estimates for the function $a(\epsilon t, \epsilon)$ where $a(T, \epsilon)$ is displayed in (21) for $\epsilon$ and $t$ in suitable domains.

Lemma 9. For any given $\delta>0$, one can find a small enough radius $\epsilon_{0}>0$ such that the next factorization

$$
a(\epsilon t, \epsilon)=-\frac{1}{\epsilon t}\left(1+a_{\text {out }}(\epsilon, t)\right)
$$


holds, provided that $\epsilon \in D\left(0, \epsilon_{0}\right) \backslash\{0\}, t \in \mathcal{A}$, where $a_{\text {out }}(\epsilon, t)$ represents a holomorphic function on $D\left(0, \epsilon_{0}\right) \times \mathcal{A}$ such that

$$
\sup _{\epsilon \in D\left(0, \epsilon_{0}\right), t \in \mathcal{A}}\left|a_{\text {out }}(\epsilon, t)\right|<\delta
$$

Proof. For $t \in \mathcal{A}$ and $\epsilon \in D\left(0, \epsilon_{0}\right) \backslash\{0\}$, we can write

$$
a(\epsilon t, \epsilon)=\frac{1}{2 \epsilon^{\frac{\alpha+1}{2}}} \log \left(\frac{\epsilon t-\epsilon^{\frac{\alpha+1}{2}}}{\epsilon t+\epsilon^{\frac{\alpha+1}{2}}}\right)=\frac{1}{2 \epsilon^{\frac{\alpha+1}{2}}} \log \left(\frac{1-\epsilon^{\frac{\alpha-1}{2}} / t}{1+\epsilon^{\frac{\alpha-1}{2}} / t}\right)
$$

By classical Taylor expansions, we can expand

$$
\log \left(\frac{1-z}{1+z}\right)=-2 z+z \varepsilon(z)
$$

for some holomorphic function $\varepsilon(z)$ near the origin such that $\lim _{z \rightarrow 0} \varepsilon(z)=0$. As a result, we can write

$$
a(\epsilon t, \epsilon)=-\frac{1}{\epsilon t}\left(1-\frac{1}{2} \varepsilon\left(\epsilon^{\frac{\alpha-1}{2}} / t\right)\right)
$$

that gives rise to the factorization (66) with the bounds (67) whenever $\epsilon_{0}$ is taken close enough to 0 .

In the sequel, we define the notion of admissible set of sectors accordingly to a good covering.

Definition 5. We consider a good covering $\underline{\mathcal{E}}^{\text {out }}=\left\{\mathcal{E}_{p}^{\text {out }}\right\}_{0 \leq p \leq l-1}$ in $\mathbb{C}^{*}$, a set of unbounded sectors $S_{\mathfrak{d}_{p}^{\text {out }}}$, $0 \leq p \leq \iota-1$, with bisecting direction $\mathfrak{d}_{p}^{\text {out }} \in \mathbb{R}$ and a disc $D(0, \rho)$ for some radius $\rho>0$ that suffer the next two conditions:

(1) A constant $\mathfrak{q}>0$ can be taken with lower bounds (49)

$$
\left|\tau-q_{l}(m)\right| \geq \mathfrak{q}(1+|\tau|)
$$

for all $0 \leq l \leq \delta_{D}-1$, all $0 \leq p \leq \iota-1$, all $m \in \mathbb{R}$, provided that $\tau \in S_{\mathfrak{d}_{p}^{\text {out }}} \cup D(0, \rho)$.

(2) We can find a constant $\Delta^{\text {out }}>0$ such that for all $\epsilon \in \mathcal{E}_{p}^{\text {out }}$, all $t \in \mathcal{A}$, a direction $\gamma_{p}^{\text {out }} \in \mathbb{R}$ (that may rely on $\epsilon, t)$ with $L_{\gamma_{p}^{\text {out }}}=\mathbb{R}_{+} e^{\sqrt{-1} \gamma_{p}^{\text {out }}} \subset S_{\mathfrak{d}_{p}^{\text {out }}} \cup\{0\}$ can be singled out for which

$$
\cos (\arg (\tau)-\arg (\epsilon)-\arg (t))>\Delta^{\text {out }}
$$

whenever $\tau \in L_{\gamma_{p}^{\text {out }}}, \epsilon \in \mathcal{E}_{p}^{\text {out }}$ and $t \in \mathcal{A}$, for all $0 \leq p \leq \iota-1$.

Then, the family of sectors

$$
\mathcal{S}^{\text {out }}=\left\{\left\{S_{\mathfrak{d}_{p}^{\text {out }}}\right\}_{0 \leq p \leq l-1}, D(0, \rho)\right\}
$$

is called admissible accordingly to the good covering $\underline{\mathcal{E}}^{\text {out }}$.

In the next first principal statement of the paper, we construct a family of so-called outer holomorphic solutions to our main Equation (11) defined on the sectors $\mathcal{E}_{p}^{\text {out }}$ of a good covering $\underline{\mathcal{E}}^{\text {out }}$ in $\mathbb{C}^{*}$ relatively to the perturbation parameter $\epsilon$. The difference between neighboring solutions are also estimated and gives rise to exponential flat bounds of order 1.

Theorem 1. Take for granted that all the above hypotheses (10), (12), (15), (21), (47), (51) hold. We select a good covering $\underline{\mathcal{E}}^{\text {out }}=\left\{\mathcal{E}_{p}^{\text {out }}\right\}_{0 \leq p \leq l-1}$ in $\mathbb{C}^{*}$ and an admissible set of sectors $\mathcal{S}^{\text {out }}=\left\{\left\{S_{\mathfrak{d}_{p}^{\text {out }}}\right\}_{0 \leq p \leq l-1}, D(0, \rho)\right\}$ accordingly to $\underline{\mathcal{E}}^{\text {out }}$.

Then, if the constants $\epsilon_{0}>0$ and $\left|c_{1,2}\right|, C_{l, 1}, D_{1}$ for $0 \leq l \leq D-1$ defined in Section 2.2 are close enough to 0 , for each $0 \leq p \leq \iota-1$, one can manufacture a solution $(t, z, \epsilon) \mapsto u_{p}^{\text {out }}(t, z, \epsilon)$ of the main equation (11) 
that is bounded holomorphic on the domain $\mathcal{A} \times H_{\beta^{\prime}} \times \mathcal{E}_{p}^{\text {out }}$ for any given $0<\beta^{\prime}<\beta$. This solution possesses a representation as special Laplace transform and Fourier integral

$$
u_{p}^{\text {out }}(t, z, \epsilon)=\frac{1}{(2 \pi)^{1 / 2}} \int_{-\infty}^{+\infty} \int_{L_{\gamma_{p}^{\text {out }}}} W_{p}^{\text {out }}(\tau, m, \epsilon) \exp (\tau a(\epsilon t, \epsilon)) e^{i z m} \frac{d \tau}{\tau} d m
$$

for a function $(\tau, m) \mapsto W_{p}^{\text {out }}(\tau, m, \epsilon)$ that appertains to the Banach space $E_{(\nu, \beta, \mu)}^{\mathfrak{o}_{p}^{\text {out }}}$ for all $\epsilon \in \mathcal{E}_{p}^{\text {out }}$, for the sector $S_{\mathfrak{d}_{p}^{\text {out }}}$ and the disc $D(0, \rho)$ taken as in Definition 5.

In addition, neighboring solutions are subjected to the next bounds: there exist constants $K_{p}^{\text {out }}, M_{p}^{\text {out }}>0$ with

$$
\sup _{t \in \mathcal{A}, z \in H_{\beta^{\prime}}}\left|u_{p+1}^{\text {out }}(t, z, \epsilon)-u_{p}^{\text {out }}(t, z, \epsilon)\right| \leq K_{p}^{\text {out }} \exp \left(-\frac{M_{p}^{\text {out }}}{|\epsilon|}\right)
$$

for all $\epsilon \in \mathcal{E}_{p+1}^{\text {out }} \cap \mathcal{E}_{p}^{\text {out }}$, for $0 \leq p \leq \iota-1$ (by convention $\left.u_{\iota}^{\text {out }}=u_{0}^{\text {out }}\right)$

Proof. Owing to the hypotheses listed above, Proposition 1 allows, for each $0 \leq p \leq \iota-1$, the existence of a solution $(\tau, m) \mapsto W_{p}^{\text {out }}(\tau, m, \epsilon)$ of the convolution Equation (29) on the domain $\left(S_{\mathfrak{d}_{p}^{\text {out }}} \cup D(0, \rho)\right) \times$ $\mathbb{R}$ in the space $E_{(v, \beta, \mu)}^{\mathfrak{d}_{p}^{\text {out }}}$ constricted to the bounds

$$
\sup _{\epsilon \in D\left(0, \epsilon_{0}\right)}\left\|W_{p}^{\text {out }}(\tau, m, \epsilon)\right\|_{(\nu, \beta, \mu)} \leq \omega_{p}^{\text {out }}
$$

for some real number $\omega_{p}^{\text {out }}>0$. For each $0 \leq p \leq \iota-1$, let us introduce the function

$$
U_{p}^{\text {out }}(T, z, \epsilon)=\frac{1}{(2 \pi)^{1 / 2}} \int_{-\infty}^{+\infty} \int_{L_{\gamma_{p}^{\text {out }}}} W_{p}^{\text {out }}(\tau, m, \epsilon) \exp (\tau a(T, \epsilon)) e^{i z m} \frac{d \tau}{\tau} d m
$$

along the halfline $L_{\gamma_{p}^{\text {out }}}=\mathbb{R}_{+} e^{\sqrt{-1} \gamma_{p}^{\text {out }}} \subset S_{\mathfrak{d}_{p}^{\text {out }}} \cup\{0\}$ singled out in (69). With the help of (72) and Lemma 9, the requirement (69) implies that for all $\epsilon \in \mathcal{E}_{p}^{\text {out }}$, the map $(T, z) \mapsto U_{p}^{\text {out }}(T, z, \epsilon)$ is bounded holomorphic on the domain

$$
\left(\{\epsilon t / t \in \mathcal{A}\} \cap D\left(0, \frac{(1-\delta) \Delta_{\text {out }}}{\left(v+\frac{1}{2}\right)}\right)\right) \times H_{\beta^{\prime}}
$$

for some tiny $\delta>0$, provided that the radius $\epsilon_{0}>0$ is chosen small enough, for any given $0<\beta^{\prime}<\beta$.

By following backwards the computations of Section 2.3 and departing from the convolution problem (29), we notice that the application $(T, z) \mapsto U_{p}^{\text {out }}(T, z, \epsilon)$ solves Equation (18) on the domain (73), for $\epsilon \in \mathcal{E}_{p}^{\text {out }}$. For $0 \leq p \leq \iota-1$, we define

$$
u_{p}^{\text {out }}(t, z, \epsilon)=U_{p}^{\text {out }}(\epsilon t, z, \epsilon)=\frac{1}{(2 \pi)^{1 / 2}} \int_{-\infty}^{+\infty} \int_{L_{\gamma_{p}^{\text {out }}}} W_{p}^{\text {out }}(\tau, m, \epsilon) \exp (\tau a(\epsilon t, \epsilon)) e^{i z m} \frac{d \tau}{\tau} d m
$$

As a consequence of the above discussion, we check that the function $(t, z, \epsilon) \mapsto u_{p}^{\text {out }}(t, z, \epsilon)$ represents a bounded holomorphic function and solves our main Equation (11) on the domain $\mathcal{A} \times H_{\beta^{\prime}} \times \mathcal{E}_{p}^{\text {out }}$ under the requirement that the radius $\epsilon_{0}$ is chosen close enough to 0.

In the last part of the proof, we turn our attention to the bounds (71). Let $0 \leq p \leq \iota-1$. The partial maps $\tau \mapsto W_{j}^{\text {out }}(\tau, m, \epsilon)$ for $j=p, p+1$ are analytic continuations on the unbounded sectors $S_{\mathfrak{d}_{p}^{\text {out }}}, S_{\mathfrak{d}_{p+1}^{\text {out }}}$ of a common analytic function that we call $\tau \mapsto W(\tau, m, \epsilon)$ on $D(0, \rho)$, for all $m \in \mathbb{R}$, all $\epsilon \in D\left(0, \epsilon_{0}\right)$. Moreover, the map $\tau \mapsto W(\tau, m, \epsilon) \exp (\tau a(\epsilon t, \epsilon)) / \tau$ is bounded holomorphic on 
$D(0, \rho)$ and by Cauchy's theorem, its integral vanishes along the oriented path described as the union of

(a) the segment joining 0 and $(\rho / 2) \exp \left(\sqrt{-1} \gamma_{p+1}^{\text {out }}\right)$,

(b) the arc of circle with radius $\rho / 2$ that links $(\rho / 2) \exp \left(\sqrt{-1} \gamma_{p+1}^{\text {out }}\right)$ and $(\rho / 2) \exp \left(\sqrt{-1} \gamma_{p}^{\text {out }}\right)$,

(c) the segment connecting $(\rho / 2) \exp \left(\sqrt{-1} \gamma_{p}^{\text {out }}\right)$ and 0 .

As a result, we can recast the difference $u_{p+1}^{\text {out }}-u_{p}^{\text {out }}$ as a sum of three integrals

$$
\begin{aligned}
& u_{p+1}^{\text {out }}(t, z, \epsilon)-u_{p}^{\text {out }}(t, z, \epsilon) \\
& =\frac{1}{(2 \pi)^{1 / 2}} \int_{-\infty}^{+\infty} \int_{L_{\rho / 2, \gamma_{p+1}^{\text {out }}}} W_{p+1}^{\text {out }}(\tau, m, \epsilon) \exp (\tau a(\epsilon t, \epsilon)) e^{i z m} \frac{d \tau}{\tau} d m \\
& -\frac{1}{(2 \pi)^{1 / 2}} \int_{-\infty}^{+\infty} \int_{L_{\rho / 2, \gamma_{p}^{\text {out }}}} W_{p}^{\text {out }}(\tau, m, \epsilon) \exp (\tau a(\epsilon t, \epsilon)) e^{i z m} \frac{d \tau}{\tau} d m \\
& +\frac{1}{(2 \pi)^{1 / 2}} \int_{-\infty}^{+\infty} \int_{C_{\rho / 2, \gamma_{p}^{\text {out }}, \gamma_{p+1}^{\text {out }}}} W(\tau, m, \epsilon) \exp (\tau a(\epsilon t, \epsilon)) e^{i z m} \frac{d \tau}{\tau} d m
\end{aligned}
$$

where

$$
L_{\rho / 2, \gamma_{j}^{\text {out }}}=[\rho / 2,+\infty) \exp \left(\sqrt{-1} \gamma_{j}^{\text {out }}\right), j=p, p+1
$$

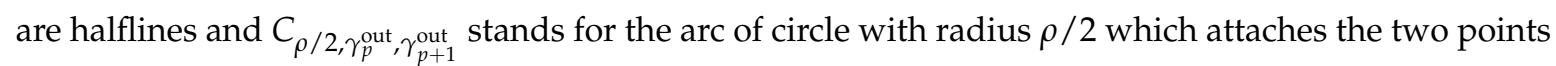
$(\rho / 2) \exp \left(\sqrt{-1} \gamma_{p}^{\text {out }}\right)$ and $(\rho / 2) \exp \left(\sqrt{-1} \gamma_{p+1}^{\text {out }}\right)$.

We first control the quantity

$$
I_{1}=\left|\frac{1}{(2 \pi)^{1 / 2}} \int_{-\infty}^{+\infty} \int_{L_{\rho / 2, \gamma_{p+1}^{\text {out }}}} W_{p+1}^{\text {out }}(\tau, m, \epsilon) \exp (\tau a(\epsilon t, \epsilon)) e^{i z m} \frac{d \tau}{\tau} d m\right|
$$

According to the condition (2) of Definition 5, bearing in mind the bounds (72) and the factorization (66), provided that the constant $\delta>0$ in (67) is taken close enough to 0 , we obtain the estimates

$$
\begin{gathered}
I_{1} \leq \frac{1}{(2 \pi)^{1 / 2}} \int_{-\infty}^{+\infty} \int_{\rho / 2}^{+\infty} \omega_{p+1}^{\text {out }}(1+|m|)^{-\mu} e^{-\beta|m|} \frac{r}{1+r^{2}} e^{\nu r} \\
\times \exp \left(-\frac{r}{|\epsilon t|}\left|1+a_{\text {out }}(\epsilon, t)\right| \cos \left(\gamma_{p+1}^{\text {out }}-\arg (\epsilon)-\arg (t)+\arg \left(1+a_{\text {out }}(\epsilon, t)\right)\right)\right) e^{-m \operatorname{Im}(z)} \frac{d r}{r} d m \\
\quad \leq \frac{\omega_{p+1}^{\text {out }}}{(2 \pi)^{1 / 2}} \int_{-\infty}^{+\infty} e^{-\left(\beta-\beta^{\prime}\right)|m|} d m \int_{\rho / 2}^{+\infty} \exp \left(-\left(\frac{(1-\delta) \Delta^{\text {out }}}{|t|}-v|\epsilon|\right) \frac{r}{|\epsilon|}\right) d r \\
\quad \leq \frac{2 \omega_{p+1}^{\text {out }}}{(2 \pi)^{1 / 2}\left(\beta-\beta^{\prime}\right)} \int_{\rho / 2}^{+\infty} \exp \left(-\frac{\delta_{2} r}{|\epsilon|}\right) d r=\frac{2 \omega_{p+1}^{\text {out }}}{(2 \pi)^{1 / 2}\left(\beta-\beta^{\prime}\right)} \frac{|\epsilon|}{\delta_{2}} \exp \left(-\frac{\rho \delta_{2}}{2|\epsilon|}\right)
\end{gathered}
$$

for some $\delta_{2}>0$ chosen under the condition that

$$
\frac{(1-\delta) \Delta^{\text {out }}}{\rho_{2}}-v \epsilon_{0}>\delta_{2}
$$

holds, for all $z \in H_{\beta^{\prime}}$, all $t \in \mathcal{A}$ and $\epsilon \in \mathcal{E}_{p}^{\text {out }} \cap \mathcal{E}_{p+1}^{\text {out }}$. 
In a similar manner, we can display upper bounds for the second integral piece

$$
I_{2}=\left|\frac{1}{(2 \pi)^{1 / 2}} \int_{-\infty}^{+\infty} \int_{L_{\rho / 2, \gamma_{p}^{\text {out }}}} W_{p}^{\text {out }}(\tau, m, \epsilon) \exp (\tau a(\epsilon t, \epsilon)) e^{i z m} \frac{d \tau}{\tau} d m\right|
$$

namely

$$
I_{2} \leq \frac{2 \omega_{p}^{\text {out }}}{(2 \pi)^{1 / 2}\left(\beta-\beta^{\prime}\right)} \frac{|\epsilon|}{\delta_{2}} \exp \left(-\frac{\rho \delta_{2}}{2|\epsilon|}\right)
$$

for some $\delta_{2}>0$ taken in a way that (78) holds, provided that $z \in H_{\beta^{\prime}}, t \in \mathcal{A}$ and $\epsilon \in \mathcal{E}_{p}^{\text {out }} \cap \mathcal{E}_{p+1}^{\text {out }}$.

In the last step, we handle the integral part along the arc of circle

$$
I_{3}=\left|\frac{1}{(2 \pi)^{1 / 2}} \int_{-\infty}^{+\infty} \int_{C_{\rho / 2, \gamma_{p} \text { ut }, \gamma_{p+1}^{\text {out }}}} W(\tau, m, \epsilon) \exp (\tau a(\epsilon t, \epsilon)) e^{i z m} \frac{d \tau}{\tau} d m\right|
$$

By construction, under the condition (69) for the directions $\gamma_{p}^{\text {out }}, \gamma_{p+1}^{\text {out }}$, we observe that the next inequality

$$
\cos (\theta-\arg (\epsilon)-\arg (t))>\Delta^{\text {out }}
$$

must hold whenever $\theta \in\left[\gamma_{p}^{\text {out }}, \gamma_{p+1}^{\text {out }}\right]$ (if $\gamma_{p}^{\text {out }}<\gamma_{p+1}^{\text {out }}$ ) or $\theta \in\left[\gamma_{p+1}^{\text {out }}, \gamma_{p}^{\text {out }}\right]$ (if $\gamma_{p+1}^{\text {out }}<\gamma_{p}^{\text {out }}$ ) for all $\epsilon \in \mathcal{E}_{p}^{\text {out }} \cap \mathcal{E}_{p+1}^{\text {out }}, t \in \mathcal{A}$. Taking into account the bounds (72) and the factorization (66), if the constant $\delta>0$ in (67) is chosen small enough, we get

$$
\begin{aligned}
I_{3} \leq & \frac{1}{(2 \pi)^{1 / 2}} \int_{-\infty}^{+\infty} \mid \int_{\gamma_{p}}^{\gamma_{p+1}} \max _{0 \leq p \leq l-1} \omega_{p}^{\text {out }}(1+|m|)^{-\mu} e^{-\beta|m|} \frac{\rho / 2}{1+(\rho / 2)^{2}} e^{\nu \rho / 2} \\
& \times \exp \left(-\frac{\rho / 2}{|\epsilon t|}\left|1+a_{\text {out }}(\epsilon, t)\right| \cos \left(\theta-\arg (\epsilon)-\arg (t)+\arg \left(1+a_{\text {out }}(\epsilon, t)\right)\right)\right) e^{-m \operatorname{Im}(z)} d \theta \mid d m \\
& \leq \frac{1}{(2 \pi)^{1 / 2}} \int_{-\infty}^{+\infty} e^{-\left(\beta-\beta^{\prime}\right)|m|} d m \frac{\rho}{2}\left|\gamma_{p+1}-\gamma_{p}\right| \max _{0 \leq p \leq l-1} \omega_{p}^{\text {out }} \exp \left(-\left(\frac{(1-\delta) \Delta^{\text {out }}}{|t|}-v|\epsilon|\right) \frac{\rho}{2|\epsilon|}\right) \\
& \leq \frac{\max _{0 \leq p \leq l-1} \omega_{p}^{\text {out }}}{(2 \pi)^{1 / 2}\left(\beta-\beta^{\prime}\right)} \rho\left|\gamma_{p+1}-\gamma_{p}\right| \exp \left(-\frac{\delta_{2} \rho}{2|\epsilon|}\right)
\end{aligned}
$$

for a well-chosen $\delta_{2}>0$ submitted to (78), whenever $z \in H_{\beta^{\prime}}, t \in \mathcal{A}$ and $\epsilon \in \mathcal{E}_{p}^{\text {out }} \cap \mathcal{E}_{p+1}^{\text {out }}$.

In conclusion, gathering the bounds (77), (79) and (80) above, we obtain the expected estimates (71) from the decomposition (75).

\subsection{Inner Solutions}

We fix a bounded sectorial annulus

$$
\chi=\left\{x \in \mathbb{C} / r_{1}<|x|<r_{2}, \alpha_{1}<\arg (x)<\alpha_{2}\right\}
$$

for some radii $r_{1}, r_{2}>0$ and angles $\alpha_{1}<\alpha_{2}$ and we define the next open sectorial domain

$$
\mathcal{T}_{\epsilon}=\left\{\epsilon^{\frac{\alpha-1}{2}} x / x \in \chi\right\}
$$

for all $\epsilon \in \mathbb{C}^{*}$.

We start this subsection with a lemma that provides bounds estimates for the function $a(\epsilon t, \epsilon)$ where $a(T, \epsilon)$ is given by (21) for $\epsilon$ and $t$ in appropriate domains. 
Lemma 10. For any fixed $\delta>0$, we can find a large enough inner radius $r_{1}>0$ such that we can factorize

$$
a(\epsilon t, \epsilon)=-\frac{1}{\epsilon^{\frac{\alpha+1}{2}} x}\left(1+a_{\text {in }}(x)\right)
$$

provided that $\epsilon \in D\left(0, \epsilon_{0}\right) \backslash\{0\}, t \in \mathcal{T}_{\epsilon}$, where $a_{\mathrm{in}}(s)$ is a holomorphic function on $\chi$ such that

$$
\sup _{s \in \chi}\left|a_{\text {in }}(s)\right| \leq \delta
$$

Proof. When $t=\epsilon^{\frac{\alpha-1}{2}} x$, we can write

$$
a(\epsilon t, \epsilon)=a\left(\epsilon^{\frac{\alpha+1}{2}} x, \epsilon\right)=\frac{1}{2 \epsilon^{\frac{\alpha+1}{2}}} \log \left(\frac{x-1}{x+1}\right)=\frac{1}{2 \epsilon^{\frac{\alpha+1}{2}}} \log \left(\frac{1-\frac{1}{x}}{1+\frac{1}{x}}\right)
$$

Then, owing to (68), we can write

$$
a(\epsilon t, \epsilon)=-\frac{1}{\epsilon^{\frac{\alpha+1}{2}} x}\left(1-\frac{1}{2} \varepsilon(1 / x)\right)
$$

from which (82) together with the bounds (83) follow.

In the next definition, we introduce the notion of admissible set of sectors relatively to a good covering.

Definition 6. We consider a good covering $\underline{\mathcal{E}}^{\text {in }}=\left\{\mathcal{E}_{p}^{\text {in }}\right\}_{0 \leq p \leq \eta-1}$ in $\mathbb{C}^{*}$, a set of unbounded sectors $S_{\mathfrak{d}_{p}^{\text {in }}}$, $0 \leq p \leq \eta-1$ with bisecting direction $\mathfrak{d}_{p}^{\text {in }} \in \mathbb{R}$ and a disc $D(0, \rho)$ for some radius $\rho>0$ that fulfill the next two of properties:

(1) A constant q can be chosen with lower bounds (49)

$$
\left|\tau-q_{l}(m)\right| \geq \mathfrak{q}(1+|\tau|)
$$

for all $0 \leq l \leq \delta_{D}-1$, all $0 \leq p \leq \eta-1$, all $m \in \mathbb{R}$, whenever $\tau \in S_{\mathfrak{d}_{p}^{\text {in }}} \cup D(0, \rho)$.

(2) There exists $\Delta^{\text {in }}>0$ such that for all $\epsilon \in \mathcal{E}_{p}^{\text {in }}$, all $t \in \mathcal{T}_{\epsilon}$, one can choose a direction $\gamma_{p}^{\text {in }} \in \mathbb{R}$ (that may depend on $\epsilon$ ) with $L_{\gamma_{p}^{\text {in }}}=\mathbb{R}_{+} e^{\sqrt{-1}} \gamma_{p}^{\text {in }} \subset S_{\mathfrak{d}_{p}^{\text {in }}} \cup\{0\}$ for which

$$
\cos \left(\arg (\tau)-\arg \left(\epsilon^{\frac{\alpha+1}{2}}\right)-\arg (x)\right)>\Delta^{\text {in }}
$$

provided that $\tau \in L_{\gamma_{p}^{\text {in }}}$ and $t=\epsilon^{\frac{\alpha-1}{2}} x \in \mathcal{T}_{\epsilon}$, whenever $0 \leq p \leq \eta-1$.

If the conditions above are both satisfied, the collection of sectors

$$
\mathcal{S}^{\text {in }}=\left\{\left\{S_{\mathfrak{d}_{p}^{\text {in }}}\right\}_{0 \leq p \leq \eta-1}, D(0, \rho)\right\}
$$

is called admissible relatively to the good covering $\underline{\mathcal{E}}^{\text {in }}$.

In the next second main statement of the work, we exhibit a collection of actual holomorphic solutions, called inner solutions, to our main Equation (11) defined on the sectors $\mathcal{E}_{p}^{\text {in }}$ of a good covering $\underline{\mathcal{E}}^{\text {in }}=\left\{\mathcal{E}_{p}^{\text {in }}\right\}_{0 \leq p \leq \eta-1}$ in $\mathbb{C}^{*}$ with respect to the perturbation parameter $\epsilon$. We control also the difference between consecutive solutions on the intersection of sectors $\mathcal{E}_{p}^{\text {in }} \cap \mathcal{E}_{p+1}^{\text {in }}$ where exponentially flat bounds leaning on $\alpha$ are observed. 
Theorem 2. Assume that the list of constraints (10), (12), (15), (21), (47), (51) hold. We consider a good covering $\underline{\mathcal{E}}^{\text {in }}=\left\{\mathcal{E}_{p}^{\text {in }}\right\}_{0 \leq p \leq \eta-1}$ in $\mathbb{C}^{*}$ and an admissible set of sectors $\mathcal{S}^{\text {in }}=\left\{\left\{S_{\mathfrak{d}_{p}^{\text {in }}}\right\}_{0 \leq p \leq \eta-1}, D(0, \rho)\right\}$ relatively to $\underline{\mathcal{E}}^{\text {in }}$.

Then, taking for granted that the constants $\epsilon_{0}>0$ and $\left|c_{1,2}\right|, C_{l, 1}, D_{1}$, for $0 \leq l \leq D-1$, introduced in the Section 2.2 are taken small enough, for each $0 \leq p \leq \eta-1$, and all $\epsilon \in \mathcal{E}_{p}^{\text {in }}$, one can construct a solution $(t, z) \mapsto u_{p}^{\text {in }}(t, z, \epsilon)$ of the main Equation (11) that is bounded holomorphic on the domain $\mathcal{T}_{\epsilon} \times H_{\beta^{\prime}}$ for any given $0<\beta^{\prime}<\beta$. This solution is represented as a special Laplace transform

$$
u_{p}^{\mathrm{in}}(t, z, \epsilon)=\frac{1}{(2 \pi)^{1 / 2}} \int_{-\infty}^{+\infty} \int_{L_{\gamma_{p}}^{\text {in }}} W_{p}^{\text {in }}(\tau, m, \epsilon) \exp (\tau a(\epsilon t, \epsilon)) e^{i z m} \frac{d \tau}{\tau} d m
$$

where the function $(\tau, m) \mapsto W_{p}^{\text {in }}(\tau, m, \epsilon)$ belongs to the Banach space $E_{(v, \beta, \mu)}^{\mathfrak{d}_{p}^{\text {in }}}$ for all $\epsilon \in \mathcal{E}_{p}^{\text {in }}$, for the sector $S_{\mathfrak{d}_{p}^{\text {in }}}$ and the disc $D(0, \rho)$ chosen according to Definition 6.

Furthermore, the functions

$$
(x, z, \epsilon) \mapsto u_{p}^{\text {in }}\left(\epsilon^{\frac{\alpha-1}{2}} x, z, \epsilon\right)
$$

are bounded holomorphic on the domain $\chi \times H_{\beta^{\prime}} \times \mathcal{E}_{p}^{\text {in }}$ for any given $0<\beta^{\prime}<\beta$ and $0 \leq p \leq \eta-1$. These functions are submitted to the next bounds: there exist constants $K_{p}^{\text {in }}, M_{p}^{\text {in }}>0$ such that

$$
\sup _{x \in \chi, z \in H_{\beta^{\prime}}}\left|u_{p+1}^{\text {in }}\left(\epsilon^{\frac{\alpha-1}{2}} x, z, \epsilon\right)-u_{p}^{\text {in }}\left(\epsilon^{\frac{\alpha-1}{2}} x, z, \epsilon\right)\right| \leq K_{p}^{\text {in }} \exp \left(-\frac{M_{p}^{\text {in }}}{|\epsilon|^{\frac{\alpha+1}{2}}}\right)
$$

for all $\epsilon \in \mathcal{E}_{p+1}^{\text {in }} \cap \mathcal{E}_{p}^{\text {in }}$, for $0 \leq p \leq \eta-1$ (where by convention $u_{\eta}^{\text {in }}=u_{0}^{\text {in }}$ ).

Proof. Under the hypotheses of Theorem 2, for each $0 \leq p \leq \eta-1$, according to Proposition 1, one can build up a solution $(\tau, m) \mapsto W_{p}^{\text {in }}(\tau, m, \epsilon)$ of the convolution Equation (29) on the domain $\left(S_{\mathfrak{d}_{p}^{\text {in }}} \cup D(0, \rho)\right) \times \mathbb{R}$ that belongs to the space $E_{(v, \beta, \mu)}^{\mathfrak{d}_{p}^{\text {in }}}$ contrived to the bounds

$$
\sup _{\epsilon \in D\left(0, \epsilon_{0}\right)}\left\|W_{p}^{\text {in }}(\tau, m, \epsilon)\right\|_{(\nu, \beta, \mu)} \leq \omega_{p}^{\text {in }}
$$

for some real number $\omega_{p}^{\text {in }}>0$. For each $0 \leq p \leq \eta-1$, we define a function

$$
U_{p}^{\text {in }}(T, z, \epsilon)=\frac{1}{(2 \pi)^{1 / 2}} \int_{-\infty}^{+\infty} \int_{L_{\gamma_{p}^{\text {in }}}} W_{p}^{\text {in }}(\tau, m, \epsilon) \exp (\tau a(T, \epsilon)) e^{i z m} \frac{d \tau}{\tau} d m
$$

where the halfline $L_{\gamma_{p}^{\text {in }}}=\mathbb{R}_{+} e^{\sqrt{-1}} \gamma_{p}^{\text {in }} \subset S_{\mathfrak{d}_{p}^{\text {in }}} \cup\{0\}$ is chosen as in (84). Owing to (88) and Lemma 10, for all $\epsilon \in \mathcal{E}_{p}^{\text {in }}$, the condition (84) allows the map $(T, z) \mapsto U_{p}^{\text {in }}(T, z, \epsilon)$ to be well defined and bounded holomorphic on the domain

$$
\left(\epsilon \mathcal{T}_{\epsilon} \cap D\left(0, \frac{(1-\delta) \Delta^{\text {in }}}{v+\frac{1}{2}}\right)\right) \times H_{\beta^{\prime}}
$$

for some small $\delta>0$ and $\Delta^{\text {in }}$ appearing in (84), provided that the inner radius $r_{1}>0$ of $\chi$ is taken large enough, for any given $0<\beta^{\prime}<\beta$.

By performing rearwards, the full record of computations of Section 2.3, we obtain that the application $(T, z) \mapsto \mathcal{U}_{p}^{\text {in }}(T, z, \epsilon)$ solves the Equation (18) on the domain (89) whenever $\epsilon \in \mathcal{E}_{p}^{\text {in }}$. At last, for all $0 \leq p \leq \eta-1$, we set

$$
u_{p}^{\text {in }}(t, z, \epsilon)=U_{p}^{\text {in }}(\epsilon t, z, \epsilon)=\frac{1}{(2 \pi)^{1 / 2}} \int_{-\infty}^{+\infty} \int_{L_{\gamma_{p}}^{\text {in }}} W_{p}^{\text {in }}(\tau, m, \epsilon) \exp (\tau a(\epsilon t, \epsilon)) e^{i z m} \frac{d \tau}{\tau} d m
$$


By the construction above, the function $(t, z) \mapsto u_{p}^{\text {in }}(t, z, \epsilon)$ is bounded and holomorphic and solves our main Equation (11) on the domain $\mathcal{T}_{\epsilon} \times H_{\beta^{\prime}}$, for all $\epsilon \in \mathcal{E}_{p}^{\text {in }}$, provided that the inner radius $r_{1}$ of $\chi$ is taken large enough and the radius $\epsilon_{0}>0$ of the disc $D\left(0, \epsilon_{0}\right)$ containing all the sectors $\mathcal{E}_{p}^{\text {in }}$ is strained to the bounds

$$
\epsilon_{0}^{\frac{\alpha+1}{2}}<\frac{(1-\delta) \Delta^{\text {in }}}{\left(v+\frac{1}{2}\right) r_{2}}
$$

where $r_{2}$ is the outer radius of $\chi$.

In the second part of the proof, we focus on the bounds (87). For the discussion and the technical content are very similar to the last part of the proof of Theorem 1 dealing with the estimates (71), the main arguments are presented in a more elliptical manner. Take $p \in\{0, \ldots, \eta-1\}$. Since the two applications $\tau \mapsto W_{j}^{\text {in }}(\tau, m, \epsilon)$, for $j=p, p+1$ are analytic continuations on the sectors $S_{\mathfrak{d}_{p}^{\text {in }}}$, $S_{\mathfrak{d}_{p+1}^{\text {in }}}$ of a joint analytic function we denote $\tau \mapsto W(\tau, m, \epsilon)$ on the disc $D(0, \rho)$, provided that $m \in \mathbb{R}$,

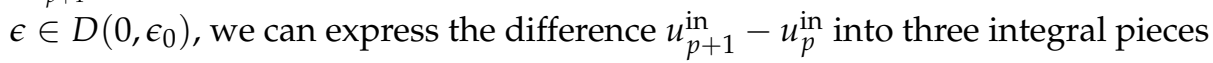

$$
\begin{aligned}
u_{p+1}^{\text {in }}\left(\epsilon^{\frac{\alpha-1}{2}} x, z, \epsilon\right) & -u_{p}^{\text {in }}\left(\epsilon^{\frac{\alpha-1}{2}} x, z, \epsilon\right) \\
= & \frac{1}{(2 \pi)^{1 / 2}} \int_{-\infty}^{+\infty} \int_{L_{\rho / 2, \gamma_{p+1}^{\text {in }}}} W_{p+1}^{\text {in }}(\tau, m, \epsilon) \exp \left(\tau a\left(\epsilon^{\frac{\alpha+1}{2}} x, \epsilon\right)\right) e^{i z m} \frac{d \tau}{\tau} d m \\
& -\frac{1}{(2 \pi)^{1 / 2}} \int_{-\infty}^{+\infty} \int_{L_{\rho / 2, \gamma_{p}^{\text {in }}}} W_{p}^{\text {in }}(\tau, m, \epsilon) \exp \left(\tau a\left(\epsilon^{\frac{\alpha+1}{2}} x, \epsilon\right)\right) e^{i z m} \frac{d \tau}{\tau} d m \\
& +\frac{1}{(2 \pi)^{1 / 2}} \int_{-\infty}^{+\infty} \int_{C_{\rho / 2, \gamma_{p}, \gamma_{p+1}^{\text {in }}}} W(\tau, m, \epsilon) \exp \left(\tau a\left(\epsilon^{\frac{\alpha+1}{2}} x, \epsilon\right)\right) e^{i z m} \frac{d \tau}{\tau} d m
\end{aligned}
$$

where $L_{\rho / 2, \gamma_{j}^{\text {in }}}, j=p, p+1$ represent halflines in directions $\gamma_{j}^{\text {in }}$ at a distance $\rho / 2$ from the origin built as in (76) and $C_{\rho / 2, \gamma_{p}^{\text {in }}, \gamma_{p+1}^{\text {in }}}$ symbolizes an arc of circle with radius $\rho / 2$ joining the above two halflines.

We evaluate the first integral block

$$
J_{1}=\left|\frac{1}{(2 \pi)^{1 / 2}} \int_{-\infty}^{+\infty} \int_{L_{\rho / 2, \gamma_{p+1}}} W_{p+1}^{\text {in }}(\tau, m, \epsilon) \exp \left(\tau a\left(\epsilon^{\frac{\alpha+1}{2}} x, \epsilon\right)\right) e^{i z m} \frac{d \tau}{\tau} d m\right|
$$

According to the condition (2) of Definition 6, bearing in mind the bounds (88) and the factorization (82), provided that the constant $\delta>0$ in (83) is selected close enough to 0 , we reach the next estimates

$$
\begin{aligned}
J_{1} \leq \frac{1}{(2 \pi)^{1 / 2}} \int_{-\infty}^{+\infty} \int_{\rho / 2}^{+\infty} \omega_{p+1}^{\text {in }}(1+|m|)^{-\mu} e^{-\beta|m|} \frac{r}{1+r^{2}} e^{v r} \\
\times \exp \left(-\frac{r}{\left|\epsilon^{\frac{\alpha+1}{2}} x\right|}\left|1+a_{i n}(x)\right| \cos \left(\gamma_{p+1}^{\text {in }}-\arg \left(\epsilon^{\frac{\alpha+1}{2}}\right)-\arg (x)+\arg \left(1+a_{i n}(x)\right)\right)\right) \\
\quad \times e^{-m \operatorname{Im}(z)} \frac{d r}{r} d m \\
\leq \frac{\omega_{p+1}^{\text {in }}}{(2 \pi)^{1 / 2}} \int_{-\infty}^{+\infty} e^{-\left(\beta-\beta^{\prime}\right)|m|} d m \int_{\rho / 2}^{+\infty} \exp \left(-\left(\frac{(1-\delta) \Delta^{\text {in }}}{|x|}-v|\epsilon|^{\frac{\alpha+1}{2}}\right) \frac{r}{|\epsilon|^{\frac{\alpha+1}{2}}}\right) d r \\
\leq \frac{2 \omega_{p+1}^{\text {in }}}{(2 \pi)^{1 / 2}\left(\beta-\beta^{\prime}\right)} \int_{\rho / 2}^{+\infty} \exp \left(-\frac{\delta_{2} r}{|\epsilon|^{\frac{\alpha+1}{2}}}\right) d r=\frac{2 \omega_{p+1}^{\text {in }}}{(2 \pi)^{1 / 2}\left(\beta-\beta^{\prime}\right)} \frac{|\epsilon|^{\frac{\alpha+1}{2}}}{\delta_{2}} \exp \left(-\frac{\delta_{2} \rho}{2|\epsilon|^{\frac{\alpha+1}{2}}}\right)
\end{aligned}
$$


for some $\delta_{2}>0$ chosen under the requirement that

$$
\frac{(1-\delta) \Delta^{\text {in }}}{r_{2}}-\delta_{2} \geq \epsilon_{0}^{\frac{\alpha+1}{2}} v
$$

holds, for all $z \in H_{\beta^{\prime}}$, all $x \in \chi$ and $\epsilon \in \mathcal{E}_{p}^{\text {in }} \cap \mathcal{E}_{p+1}^{\text {in }}$.

Likewise, we can exhibit upper bounds for the second integral component

$$
J_{2}=\left|\frac{1}{(2 \pi)^{1 / 2}} \int_{-\infty}^{+\infty} \int_{L_{\rho / 2, \gamma_{p}^{\text {in }}}} W_{p}^{\text {in }}(\tau, m, \epsilon) \exp \left(\tau a\left(\epsilon^{\frac{\alpha+1}{2}} x, \epsilon\right)\right) e^{i z m} \frac{d \tau}{\tau} d m\right|
$$

namely

$$
J_{2} \leq \frac{2 \omega_{p}^{\text {in }}}{(2 \pi)^{1 / 2}\left(\beta-\beta^{\prime}\right)} \frac{|\epsilon|^{\frac{\alpha+1}{2}}}{\delta_{2}} \exp \left(-\frac{\delta_{2} \rho}{2|\epsilon|^{\frac{\alpha+1}{2}}}\right)
$$

for some $\delta_{2}>0$ taken in a way that (93) holds, provided that $z \in H_{\beta^{\prime}}, x \in \chi$ and $\epsilon \in \mathcal{E}_{p}^{\text {in }} \cap \mathcal{E}_{p+1}^{\text {in }}$.

Lastly, we discuss the integral part along the arc of circle

$$
J_{3}=\left|\frac{1}{(2 \pi)^{1 / 2}} \int_{-\infty}^{+\infty} \int_{C_{\rho / 2, \gamma_{p}^{\text {in }}, \gamma_{p+1}^{\text {in }}}} W(\tau, m, \epsilon) \exp \left(\tau a\left(\epsilon^{\frac{\alpha+1}{2}} x, \epsilon\right)\right) e^{i z m} \frac{d \tau}{\tau} d m\right|
$$

Under the condition (84) for the directions $\gamma_{p}^{\text {in }}$ and $\gamma_{p+1}^{\text {in }}$, we check that the next inequality

$$
\cos \left(\theta-\arg \left(\epsilon^{\frac{\alpha+1}{2}}\right)-\arg (x)\right)>\Delta^{\text {in }}
$$

must hold whenever $\theta \in\left[\gamma_{p}^{\text {in }}, \gamma_{p+1}^{\text {in }}\right]$ (if $\gamma_{p}^{\text {in }}<\gamma_{p+1}^{\text {in }}$ ) or $\theta \in\left[\gamma_{p+1}^{\text {in }}, \gamma_{p}^{\text {in }}\right]$ (if $\gamma_{p+1}^{\text {in }}<\gamma_{p}^{\text {in }}$ ), for $\epsilon \in \mathcal{E}_{p}^{\text {in }} \cap \mathcal{E}_{p+1}^{\text {in }}$ and all $x \in \chi$. Taking into account the bounds (88) and the factorization (82), if the constant $\delta>0$ in (83) is chosen small enough, we get

$$
\begin{aligned}
J_{3} \leq \frac{1}{(2 \pi)^{1 / 2}} \int_{-\infty}^{+\infty} \mid \int_{\gamma_{p}^{\text {in }}}^{\gamma_{p+1}^{\text {in }}} \max _{0 \leq p \leq \eta-1} \omega_{p}^{\text {in }}(1+|m|)^{-\mu} e^{-\beta|m|} \frac{\rho / 2}{1+(\rho / 2)^{2}} e^{v \rho / 2} \\
\times \exp \left(-\frac{\rho / 2}{\left|\epsilon^{\frac{\alpha+1}{2}} x\right|}\left|1+a_{i n}(x)\right| \cos \left(\theta-\arg \left(\epsilon^{\frac{\alpha+1}{2}}\right)-\arg (x)+\arg \left(1+a_{i n}(x)\right)\right)\right) \\
\times e^{-m \operatorname{Im}(z)} d \theta\left|d m \leq \frac{1}{(2 \pi)^{1 / 2}} \int_{-\infty}^{+\infty} e^{-\left(\beta-\beta^{\prime}\right)|m|} d m \frac{\rho}{2}\right| \gamma_{p+1}^{\text {in }}-\gamma_{p}^{\text {in }} \mid \max _{0 \leq p \leq \eta-1} \omega_{p}^{\text {in }} \\
\times \exp \left(-\left(\frac{(1-\delta) \Delta^{\text {in }}}{|x|}-v|\epsilon|^{\frac{\alpha+1}{2}}\right) \frac{\rho}{2|\epsilon|^{\frac{\alpha+1}{2}}}\right) \\
\quad \leq \frac{\max _{0 \leq p \leq \eta-1} \omega_{p}^{\text {in }}}{(2 \pi)^{1 / 2}} \frac{2}{\beta-\beta^{\prime}} \frac{\rho}{2}\left|\gamma_{p+1}^{\text {in }}-\gamma_{p}^{\text {in }}\right| \exp \left(-\frac{\delta_{2} \rho}{2|\epsilon|^{\frac{\alpha+1}{2}}}\right)
\end{aligned}
$$

for a well-chosen $\delta_{2}>0$ submitted to (93) whenever $z \in H_{\beta^{\prime}}, x \in \chi$ and $\epsilon \in \mathcal{E}_{p}^{\text {in }} \cap \mathcal{E}_{p+1}^{\text {in }}$.

In conclusion, we take a radius $\epsilon_{0}>0$ that satisfies both (90) and (93). Gathering the bounds (92), (94) and (95), we obtain the expected estimates (87) from the decomposition (91).

\subsection{Parametric Asymptotic Expansions}

In the third central result of the paper, we show that the actual holomorphic outer (resp. inner) solutions to (11) obtained in Theorem 1 (resp. Theorem 2) share a common asymptotic expansion relatively to $\epsilon$ on the sectors $\mathcal{E}_{p}^{\text {out }}$ (resp. $\mathcal{E}_{p}^{\text {in }}$ ) that turns out to be of Gevrey type. 
Theorem 3. (1) Take for granted that the aforementioned requirements recorded in Theorem 1 hold. We set $\mathcal{O}_{b}\left(\mathcal{A} \times H_{\beta^{\prime}}\right)$ as the Banach space of $\mathbb{C}$-valued bounded holomorphic functions on $\mathcal{A} \times H_{\beta^{\prime}}$ equipped with the sup norm.

Then, the bounded holomorphic solutions of (11), presented in (70), considered to be maps from $\mathcal{E}_{p}^{\text {out }}$ into $\mathcal{O}_{b}\left(\mathcal{A} \times H_{\beta^{\prime}}\right)$, own a formal power series

$$
\hat{O}(\epsilon)=\sum_{k \geq 0} O_{k} \epsilon^{k} \in \mathcal{O}_{b}\left(\mathcal{A} \times H_{\beta^{\prime}}\right)[[\epsilon]]
$$

as Gevrey asymptotic expansion of order 1 . Specifically, for all $0 \leq p \leq \iota-1$, two constants $A_{p}^{\text {out }}, B_{p}^{\text {out }}>0$ can be singled out with

$$
\sup _{t \in \mathcal{A}, z \in H_{\beta^{\prime}}}\left|u_{p}^{\text {out }}(t, z, \epsilon)-\sum_{k=0}^{n-1} O_{k} \epsilon^{k}\right| \leq A_{p}^{\text {out }}\left(B_{p}^{\text {out }}\right)^{n} \Gamma(1+n)
$$

for all $n \geq 1$, provided that $\epsilon \in \mathcal{E}_{p}^{\text {out }}$. Furthermore, in the case that one sector $\mathcal{E}_{p_{0}}^{\text {out }}$, for $0 \leq p_{0} \leq \iota-1$, can be taken slightly larger than $\pi$, the map $\epsilon \mapsto\left((t, z) \mapsto u_{p_{0}}^{\text {out }}(t, z, \epsilon)\right)$ becomes the 1 -sum of the formal power series $\hat{O}(\epsilon)$ on $\mathcal{E}_{p_{0}}^{\text {out }}$, meaning that it is the unique $\mathcal{O}_{b}\left(\mathcal{A} \times H_{\beta^{\prime}}\right)$-valued holomorphic function that suffers the bounds (96) on $\mathcal{E}_{p_{0}}^{\text {out }}$.

(2) Assume that the foregoing constraints listed in Theorem 2 hold. Let us denote $\mathcal{O}_{b}\left(\chi \times H_{\beta^{\prime}}\right)$ the Banach space of $\mathbb{C}$-valued bounded holomorphic functions on $\chi \times H_{\beta^{\prime}}$ endowed with the sup norm.

Then, for all $0 \leq p \leq \eta-1$, the holomorphic and bounded functions $\epsilon \mapsto\left((x, z) \mapsto u_{p}^{\text {in }}\left(\epsilon^{\frac{\alpha-1}{2}} x, z, \epsilon\right)\right)$ built up in (86) and viewed as maps from $\mathcal{E}_{p}^{\text {in }}$ into $\mathcal{O}_{b}\left(\chi \times H_{\beta^{\prime}}\right)$, admit a formal power series

$$
\hat{I}(\epsilon)=\sum_{k \geq 0} I_{k} \epsilon^{k} \in \mathcal{O}_{b}\left(\chi \times H_{\beta^{\prime}}\right)[[\epsilon]]
$$

as Gevrey asymptotic expansion of order $\frac{2}{\alpha+1}$. This means that for all $0 \leq p \leq \eta-1$, one can find constants $A_{p}^{\text {in }}, B_{p}^{\text {in }}>0$ such that

$$
\sup _{x \in \chi, z \in H_{\beta^{\prime}}}\left|u_{p}^{\text {in }}\left(\epsilon^{\frac{\alpha-1}{2}} x, z, \epsilon\right)-\sum_{k=0}^{n-1} I_{k} \epsilon^{k}\right| \leq A_{p}^{\text {in }}\left(B_{p}^{\text {in }}\right)^{n} \Gamma\left(1+\frac{2}{\alpha+1} n\right)
$$

for all $n \geq 1$, whenever $\epsilon \in \mathcal{E}_{p}^{\text {in }}$. Moreover, if the opening of one sector $\mathcal{E}_{p_{0}}^{\text {in }}$ for a single $0 \leq p_{0} \leq \eta-1$ can be chosen a little larger than $\frac{2 \pi}{\alpha+1}$, then the map $\epsilon \mapsto\left((x, z) \mapsto u_{p_{0}}^{\text {in }}\left(\epsilon^{\frac{\alpha-1}{2}} x, z, \epsilon\right)\right)$ is elected as the $\frac{\alpha+1}{2}-$ sum of the formal series $\hat{I}(\epsilon)$ on $\mathcal{E}_{p_{0}}^{\text {in }}$ in the sense that it is the unique $\mathcal{O}_{b}\left(\chi \times H_{\beta^{\prime}}\right)$-valued holomorphic function that fulfills the inequality (97) on $\mathcal{E}_{p_{0}}^{\text {in }}$.

Proof. The proof of both parts 1) and 2) lean on a cohomological criterion for the existence of Gevrey asymptotic expansions of order $1 / k$, for real numbers $k>1 / 2$, for suitable families of sectorial holomorphic functions known as Ramis-Sibuya theorem in the literature, see ([32], p. 121) or [33], Lemma XI-2-6. Here we need a Banach valued version of this result that can be stated as follows.

Theorem 4 (Ramis-Sibuya). We set $\left(\mathbb{F},\|.\|_{\mathbb{F}}\right)$ as a Banach space over $\mathbb{C}$ and consider a good covering $\left\{\mathcal{E}_{p}\right\}_{0 \leq p \leq l-1}$ in $\mathbb{C}^{*}$. For all $0 \leq p \leq \iota-1, G_{p}$ stands for a holomorphic function from $\mathcal{E}_{p}$ into the Banach space $\left(\mathbb{F},\|.\|_{\mathbb{F}}\right)$ and let the cocycle $\Theta_{p}(\epsilon)=G_{p+1}(\epsilon)-G_{p}(\epsilon)$ be a holomorphic function from the sector $Z_{p}=$ $\mathcal{E}_{p+1} \cap \mathcal{E}_{p}$ into $\mathbb{F}$ (under the convention that $\mathcal{E}_{l}=\mathcal{E}_{0}$ and $G_{l}=G_{0}$ ). We ask for the following requirements.

(1) The functions $G_{p}(\epsilon)$ are bounded on $\mathcal{E}_{p}$, for all $0 \leq p \leq \iota-1$. 
(2) The functions $\Theta_{p}(\epsilon)$ suffer exponential flatness of order $k$ on $Z_{p}$, for all $0 \leq p \leq \imath-1$. Specifically, there exist constants $C_{p}, A_{p}>0$ such that

$$
\left\|\Theta_{p}(\epsilon)\right\|_{\mathbb{F}} \leq C_{p} e^{-A_{p} /|\epsilon|^{k}}
$$

for all $\epsilon \in Z_{p}$, all $0 \leq p \leq \iota-1$.

Then, for all $0 \leq p \leq \iota-1$, the functions $G_{p}(\epsilon)$ share a common formal power series $\hat{G}(\epsilon)=\sum_{k \geq 0} H_{k} \epsilon^{k}$ where the coefficients $H_{k}$ belong to $\mathbb{F}$, as Gevrey asymptotic expansion of order $1 / k$ on $\mathcal{E}_{p}$. In other words, constants $A_{p}, B_{p}>0$ can be selected with

$$
\left\|G_{p}(\epsilon)-\sum_{k=0}^{n-1} H_{k} \epsilon^{k}\right\|_{\mathbb{F}} \leq A_{p}\left(B_{p}\right)^{n} \Gamma\left(1+\frac{n}{k}\right)|\epsilon|^{n}
$$

for all $n \geq 1$, provided that $\epsilon \in \mathcal{E}_{p}$.

Furthermore, if one assumes that the opening of one sector $\mathcal{E}_{p_{0}}$ is slightly larger than $\pi / k$, the function $G_{p_{0}}(\epsilon)$ is promoted as a $k$-sum of $\hat{G}(\epsilon)$ on $\mathcal{E}_{p_{0}}$ which means that $G_{p_{0}}(\epsilon)$ is then the unique holomorphic function defined on $\mathcal{E}_{p_{0}}$ with the feature (98).

Regarding the first point (1), the Ramis-Sibuya theorem can be called in to the family of functions

$$
G_{p}(\epsilon):=(t, z) \mapsto u_{p}^{\text {out }}(t, z, \epsilon)
$$

for $0 \leq p \leq \imath-1$, which are bounded holomorphic functions from $\mathcal{E}_{p}^{\text {out }}$ into the Banach space $\mathbb{F}=\mathcal{O}_{b}\left(\mathcal{A} \times H_{\beta^{\prime}}\right)$ endowed with the sup norm over $\mathcal{A} \times H_{\beta^{\prime}}$. Specifically, the estimates (71) show that the cocycle $\Theta_{p}(\epsilon)=G_{p+1}(\epsilon)-G_{p}(\epsilon)$ must be restrained to the bounds (2) above for $k=1$. The existence of a formal series $\hat{O}(\epsilon)$ that conforms the statement of Theorem 3 follows.

For the second point (2), we apply the above theorem to the set of functions

$$
G_{p}(\epsilon):=(x, z) \mapsto u_{p}^{\text {in }}\left(\epsilon^{\frac{\alpha-1}{2}} x, z, \epsilon\right)
$$

for $0 \leq p \leq \eta-1$, which represent holomorphic and bounded functions from $\mathcal{E}_{p}^{\text {in }}$ into the Banach space $\mathbb{F}=\mathcal{O}_{b}\left(\chi \times H_{\beta^{\prime}}\right)$ equipped with the sup norm over $\chi \times H_{\beta^{\prime}}$. Indeed, the bounds (87) allow the cocycle $\Theta_{p}(\epsilon)=G_{p+1}(\epsilon)-G_{p}(\epsilon)$ to fulfill the constraint (2) overhead for $k=\frac{\alpha+1}{2}$. As a result, we deduce the existence of a formal power series $\hat{I}(\epsilon)$ that match the statement of Theorem 3 .

Funding: This research received no external funding.

Acknowledgments: The author thanks the University of Lille in France for its partial financial contribution for the APC of this article in the framework of the IOAP.

Conflicts of Interest: The author declares no conflict of interest.

\section{References}

1. Lastra, A.; Malek, S. Parametric Gevrey asymptotics for some nonlinear initial value Cauchy problems. J. Differ. Equ. 2015, 259, 5220-5270. [CrossRef]

2. Lope, J. Existence and uniqueness theorems for a class of linear Fuchsian partial differential equations. J. Math. Sci. Univ. Tokyo 1999, 6, 527-538.

3. Lope, J. A sharp existence and uniqueness theorem for linear Fuchsian partial differential equations. Tokyo J. Math. 2001, 24, 477-486. [CrossRef]

4. Tahara, H.; Yamane, H. Logarithmic singularities of solutions to nonlinear partial differential equations. J. Math. Soc. Jpn. 2008, 60, 603-630. [CrossRef]

5. Yamane, H. Nonlinear wave equations and singular solutions. Proc. Am. Math. Soc. 2007, 135, 3659-3667. [CrossRef] 
6. Kichenassamy, S. Fuchsian Reduction. Applications to Geometry, Cosmology, and Mathematical Physics; Progress in Nonlinear Differential Equations and their Applications, 71; Birkhäuser Boston, Inc.: Boston, MA, USA, 2007; $\mathrm{xvi}+289 \mathrm{p}$.

7. Klimes, M. Confluence of singularities of nonlinear differential equations via Borel-Laplace transformations. J. Dyn. Control Syst. 2016, 22, 285-324. [CrossRef]

8. Sternin, B.; Shatalov, V. On the confluence phenomenon for Fuchsian equations. J. Dynam. Control Syst. 1997, 3, 433-448. [CrossRef]

9. Schäfke, R. Confluence of several regular singular points into an irregular singular one. J. Dynam. Control Syst. 1998, 4, 401-424. [CrossRef]

10. Glutsyuk, A. Resonant confluence of singular points and Stokes phenomena. J. Dynam. Control Syst. 2004, 10, 253-302. [CrossRef]

11. Glutsyuk, A. Stokes operators via limit monodromy of generic perturbation. J. Dynam. Control Syst. 1999, 5, 101-135. [CrossRef]

12. Stoyanova, T. Zero level perturbation of a certain third-order linear solvable ODE with an irregular singularity at the origin of Poincaré rank 1. J. Dyn. Control Syst. 2018, 24, 511-539. [CrossRef]

13. Bolibrukh, A. On isomonodromic confluences of Fuchsian singularities. Tr. Mat. Inst. Steklova 1998, 221, 127-142; translation in Proc. Steklov Inst. Math. 1998, 221, 117-132. (In Russian)

14. Bibilo, Y. Isomonodromic confluence of singular points. (Russian) Mat. Zametki 2010, 87, 330-336; translation in Math. Notes 2010, 87, 309-315. (In Russian) [CrossRef]

15. Oshima, T. Versal Unfolding of Irregular Singularities of a Linear Differential Equation on the Riemann Sphere. Available online: https:/ / www.ms.u-tokyo.ac.jp/ oshima/paper/conf31.pdf (accessed on 20 November 2019).

16. Oshima, T. Confluence and Versal Unfolding of Pfaffian Equations. Available online: https://www.ms.utokyo.ac.jp/ oshima/paper/josai20.pdf (accessed on 20 November 2019).

17. Hurtubise, J.; Lambert, C.; Rousseau, C. Complete system of analytic invariants for unfolded differential linear systems with an irregular singularity of Poincaré rank k. Mosc. Math. J. 2014, 14, 309-338, 427. [CrossRef]

18. Hurtubise, J.; Rousseau, C. Moduli space for generic unfolded differential linear systems. Adv. Math. 2017, 307, 1268-1323. [CrossRef]

19. Lambert, C.; Rousseau, C. Complete system of analytic invariants for unfolded differential linear systems with an irregular singularity of Poincaré rank 1. Mosc. Math. J. 2012, 12, 77-138, 215. [CrossRef]

20. Lambert, C.; Rousseau, C. Moduli space of unfolded differential linear systems with an irregular singularity of Poincaré rank 1. Mosc. Math. J. 2013, 13, 529-550, 553-554. [CrossRef]

21. Klimes, M. Stokes phenomenon and confluence in non-autonomous Hamiltonian systems. Qual. Theory Dyn. Syst. 2018, 17, 665-708. [CrossRef]

22. Mozo-Fernández, J.; Schäfke, R. Asymptotic expansions and summability with respect to an analytic germ. Publ. Mat. 2019, 63, 3-79. [CrossRef]

23. Lomov, S.A. Introduction to the General Theory of Singular Perturbations; Translations of Mathematical Monographs, 112; American Mathematical Society: Providence, RI, USA, 1992; xviii+375p.

24. Fruchard, A.; Schäfke, R. Composite Asymptotic Expansions; Lecture Notes in Mathematics, 2066; Springer: Heidelberg, Germany, 2013; x+161p.

25. Ruban, A. Fluid Dynamics. Part 2. Asymptotic Problems of Fluid Dynamics; Oxford University Press: Oxford, UK, 2015; ix+319p.

26. Ruban, A. Fluid Dynamics. Part 3. Boundary Layers; Oxford University Press: Oxford, UK, 2018; xi+384p.

27. Skinner, L. Singular Perturbation Theory; Springer: New York, NY, USA, 2011; x+85p.

28. Costin, O.; Tanveer, S. Existence and uniqueness for a class of nonlinear higher-order partial differential equations in the complex plane. Commun. Pure Appl. Math. 2000, 53, 1092-1117. [CrossRef]

29. Malek, S. On Gevrey asymptotics for some nonlinear integro-differential equations. J. Dyn. Control Syst. 2010, 16, 377-406. [CrossRef]

30. Costin, O.; Tanveer, S. Short time existence and Borel summability in the Navier-Stokes equation in $\mathbb{R}^{3}$. Commun. Partial. Differ. Equ. 2009, 34, 785-817. [CrossRef]

31. O'Malley, R. Singular Perturbation Methods for Ordinary Differential Equations; Applied Mathematical Sciences, 89; Springer: New York, NY, USA, 1991; viii+225p. 
32. Balser, W. Formal Power Series and Linear Systems of Meromorphic Ordinary Differential Equations; Universitext; Springer: New York, NY, USA, 2000; xviii+299p.

33. Hsieh, P.; Sibuya, Y. Basic Theory of Ordinary Differential Equations; Universitext; Springer: New York, NY, USA, 1999.

(C) 2020 by the authors. Licensee MDPI, Basel, Switzerland. This article is an open access article distributed under the terms and conditions of the Creative Commons Attribution (CC BY) license (http://creativecommons.org/licenses/by/4.0/). 